\begin{tabular}{c|c|c|c|} 
SELECCIONES MATEMÁTICAS \\
Universidad Nacional de Trujillo \\
ISSN: $2411-1783$ (Online) \\
2021; Vol. 8(1): 100-119.
\end{tabular}

\title{
Rational Bilinear Forms as Cocycles
}

\author{
Felipe Clímaco Ccolque T. ${ }^{\circledR}$
}

Received, Set. 30, 2020

Accepted, Jan. 03, 2021

How to cite this article:

Ccolque Felipe C. Rational Bilinear Forms as Cocycles. Selecciones Matemáticas. 2021;8(1):100-119. http: //dx.doi.org/10.17268/sel.mat.2021.01.10

\begin{abstract}
In this article, some nontrivial 2-cocycles are computed for crossed products of algebraic extensions of the field of rational numbers $\mathbb{Q}$ with cyclic groups of order 2 and 3, with finite groups of order 4, 6 and 8 . The 2 -cocycles are applied for bilinear forms over finite-dimensional vector space whose scalars field is $\mathbb{Q}$ and few examples of these cocycles are presented in terms of matrices.
\end{abstract}

Keywords . Rational bilinear form, 2-cocycle, crossed product, Galois extension, Galois Group of a polynomial.

1. Introduction. The authors of [1] studied the Hochschild (co)homology of a twisted group algebra $A *_{f} G$, where $A / k$ is a simple algebraic extension of fields and $G$ is a finite groupIn this case $E=A *_{f} G$ is a crossed product of an algebra with a group, where $f$ is a $2-$ cocyclic for $E$. The study of Hochschild homology of the crossed product $E=\frac{K[X]}{\left\langle X^{2}\right\rangle} \rtimes_{f} C_{2}$ originated the concern of to compute the nontrivial cocycles of crossed products. We inquire if $k=\mathbb{Q}$ and $G=S_{3}$ (nonabelian group), is possible to find a nontrivial 2-cocycle $f$ such that $A \rtimes_{f} G$ is a crossed product for some $\mathbb{Q}$-algebra $A$.

In [2] is presented as example of polynomial not solvable by radicals $f(x)=2 x^{5}-5 x^{4}+5$ over $\mathbb{Q}$ by means of Affirmation 2: If $f(x)$ is an irreducible polynomial in $\mathbb{Q}[x]$ of degree 5 that has exactly 2 nonreal roots in the field of the complex numbers, then Galois group of $f(x)$ is the symmetric group $S_{5}$.

Following the same arguments, it is noted that this affirmation holds for polynomials of degree prime $p \geq 3$, the Galois group of an irreducible polynomial $f(x)$ in $\mathbb{Q}[x]$ of degree 3 that has exactly 2 nonreal roots in the field of the complex numbers is the symmetric group $S_{3}$. Using this fact and the characterization of a crossed product [3, Corollary 4.6] an affirmative answer is given to the question (see Section 4).

The purpose of this article is to show examples of nontrivial 2-cocycles for crossed products of the forms $\frac{\mathbb{Q}[X]}{\langle P\rangle} \rtimes_{f} C_{2}, \frac{\mathbb{Q}[X]}{\langle P\rangle} \rtimes_{f} C_{3}$ and $\frac{\mathbb{Q}[X]}{\langle P\rangle} \rtimes_{f} G_{v}$. The development of this work is done in the three sections: In the second section, a crossed product of an algebra with a group is presented.

In the third section, we apply the term of the cochain complex " 2 -cocycle" for bilinear forms over finitedimensional vector space whose scalars field is $\mathbb{Q}$ and are presented few examples of these cocycles in terms of matrices.

In the fourth section, we give examples of rational nontrivial 2-cocycles for the crossed products $\frac{\mathbb{Q}[X]}{\langle P\rangle} \rtimes_{f} C_{2}, \frac{\mathbb{Q}[X]}{\langle P\rangle} \rtimes_{f} C_{3}, \frac{\mathbb{Q}[X]}{\langle P\rangle} \rtimes_{f} G_{4}, \frac{\mathbb{Q}[X]}{\langle P\rangle} \rtimes_{f} G_{6}$ and $\frac{\mathbb{Q}[X]}{\langle P\rangle} \rtimes_{f} G_{8}$. Furthermore, it is shown that a crossed product of the form $\frac{\mathbb{Q}[X]}{\langle P\rangle} \rtimes_{f} G_{v}$ can have 2-cycles which are nonrational.

2. Cross Product of an Algebra with a Group. Let $G$ a group, $K$ a field, $A$ a $K$-algebra and $A^{*}$ the group of units of $A$. Let $\sigma: G \rightarrow A u t_{K}(A), g \mapsto \sigma(g)(a)=a^{g} \forall a \in A$, an action of $G$ on $A$; and $f: G \times G \rightarrow A^{*},(g, h) \mapsto f(g, h)$ a map .

*Facultad de Ciencias, UNI-IMCA, Calle los Biólogos, Urb San Cesar, La Molina, Lima 12-Perú. (ccolque@imca .edu •pe). 
Definition 2.1. [1] A crossed product, $A \rtimes_{f} G$, of a $K$-algebra $A$ with a group $G$, is a $K$-vector space $A \otimes K[G]$ endowed with the multiplication

$$
\left(a \omega_{g}\right)\left(b \omega_{h}\right)=a b^{g} f(g, h) \omega_{g h}
$$

satisfying the following 3 conditions :

1) $f(1, g)=f(g, 1)=1$,

2) $f\left(g_{1}, g_{2}\right)^{g_{0}} f\left(g_{0}, g_{1} g_{2}\right)=f\left(g_{0}, g_{1}\right) f\left(g_{0} g_{1}, g_{2}\right)$,

3) $\left(a^{g_{1}}\right)^{g_{0}} f\left(g_{0}, g_{1}\right)=f\left(g_{0}, g_{1}\right) a^{g_{0} g_{1}}$.

Condition 1 ) is the condition of normality, 2) is the condition of 2-cocycle and 3 ) is the twisted module condition.

Remark 2.1. $E=A \rtimes_{f} G$ is a Hopf crossed product of $A$ by $H=K[G]$.

$H=K[G]$ is a Hopf algebra with canonical basis $G$ (see [4, Example 2.2.11]), where the comultiplication $\Delta$ and counit $\varepsilon$, are given by $\Delta(g)=g \otimes g$ and $\varepsilon(g)=1, \forall g \in G$.

By definition, $A \rtimes_{f} G$ is the $K$-vector space $A \otimes K[G]$ with multiplication given by $\left(a \omega_{g}\right)\left(b \omega_{h}\right)=a b^{g} f(g, h) \omega_{g h}$ satisfying the conditions of normality, of 2-cocycle and twisted module. In terms of tensorial product $\otimes=\otimes_{K}$,

$$
\begin{aligned}
(a \otimes g)(b \otimes h) & =a b^{g} f(g, h) \otimes g h, \quad \text { since } \Delta(g)=g \otimes g, \\
& =a b^{g^{(1)}} f\left(g^{(2)}, h^{(1)}\right) \otimes g^{(3)} h^{(2)} .
\end{aligned}
$$

Since $\Delta(g)=g \otimes g$ and $\varepsilon(g)=1$, the following 3 conditions are satisfied :

1) $f(1, g)=f(g, 1)=\varepsilon(g) 1_{A}$,

2) $f\left(g_{1}{ }^{(1)}, g_{2}{ }^{(1)}\right)^{g_{0}{ }^{(1)}} f\left(g_{0}{ }^{(2)}, g_{1}{ }^{(2)} g_{2}{ }^{(2)}\right)=f\left(g_{0}{ }^{(1)}, g_{1}{ }^{(1)}\right) f\left(g_{0}{ }^{(2)} g_{1}{ }^{(2)}, g_{2}\right)$,

3) $\left(a^{g_{1}{ }^{(1)}}\right)^{g_{0}{ }^{(1)}} f\left(g_{0}{ }^{(2)}, g_{1}{ }^{(2)}\right)=f\left(g_{0}{ }^{(1)}, g_{1}{ }^{(1)}\right) a^{g_{0}{ }^{(2)} g_{1}{ }^{(2)}}$.

It is observed that the action $\sigma$ of $G$ on $A$, induces a weak action \# of $K[G]$ on $A$ through \# $(g, a)=\sigma(g)(a)$ for $g \in G$; while that the map $f: G \times G \rightarrow A^{*}$ induces a $K$-bilinear map $f: K[G] \times K[G] \rightarrow A$. Therefore, according to [5] $E=A \#{ }_{f} K[G]$; that is, $E$ is a Hopf crossed product of $A$ by $K[G]$.

3. Rational Bilinear Forms. In this section, the matrix of a rational bilinear form in an ordered basis $\mathcal{B}$ of a vector space $\mathbb{V}$ of dimension $n$ is introduced, and the concept of normal 2-cocycle over $\mathbb{V}$ is given.

Definition 3.1. Let $\mathbb{V}$ be a vector space over the field of the rational numbers $\mathbb{Q}$. A rational bilinear form on $\mathbb{V}$ is a function $f: \mathbb{V} \times \mathbb{V} \rightarrow \mathbb{Q}$, which assigns to each ordered pair of vectors $u, v \in \mathbb{V}$ a scalar $f(u, v) \in \mathbb{Q}$ such that:

i) $f\left(\lambda u_{1}+u_{2}, v\right)=\lambda, f\left(u_{1}, v\right)+f\left(u_{2}, v\right)$,

ii) $f\left(u, \lambda v_{1}+v_{2}\right)=\lambda f\left(u, v_{1}\right)+f\left(u, v_{2}\right)$,

for all $u, u_{1}, u_{2}, v, v_{1}, v_{2} \in \mathbb{V}$ and all scalar $\lambda \in \mathbb{Q}$.

Let $\mathbb{V}$ be a finite-dimensional vector space over the field $\mathbb{Q}, \operatorname{dim} \mathbb{V}=2$ and $\mathcal{B}=\left\{e_{1}, e_{2}\right\}$ an ordered basis of $\mathbb{V}$. Let $u=x_{1} e_{1}+x_{2} e_{2}$ and $v=y_{1} e_{1}+y_{2} e_{2}$ be vectors in $\mathbb{V}$.

Example 3.1. The function $f: \mathbb{V} \times \mathbb{V} \rightarrow \mathbb{Q}$ defined by

$$
f(u, v)=2 x_{1} y_{1}+3 x_{2} y_{2}
$$

is a rational bilinear form on $\mathbb{V}$.

If $u, u^{\prime}, v \in \mathbb{V}$ and $\lambda \in \mathbb{Q}$, then

$$
\begin{aligned}
f\left(\lambda u+u^{\prime}, v\right) & =2\left(\lambda x_{1}+x_{1}^{\prime}\right) y_{1}+3\left(\lambda x_{2}+x_{2}^{\prime}\right) y_{2} \\
& =\lambda\left(2 x_{1} y_{1}+3 x_{2} y_{2}\right)+2 x_{1}^{\prime} y_{1}+3 x_{2}^{\prime} y_{2} \\
& =\lambda f(u, v)+f\left(u^{\prime}, v\right)
\end{aligned}
$$

Similarly, $f\left(u, \lambda v+v^{\prime}\right)=\lambda f(u, v)+f\left(u, v^{\prime}\right)$ for all $u, v, v^{\prime} \in \mathbb{V}$ and $\lambda \in \mathbb{Q}$.

Example 3.2. The function $f: \mathbb{V} \times \mathbb{V} \rightarrow \mathbb{Q}$ defined by

$$
f(u, v)=x_{1} y_{1}+x_{1} y_{2}+x_{2} y_{1}+3 x_{2} y_{2}
$$

is a rational bilinear form on $\mathbb{V}$.

If $u, v, v^{\prime} \in \mathbb{V}$ and $\lambda \in \mathbb{Q}$, then

$$
\begin{aligned}
f\left(u, \lambda v^{\prime}+v\right) & =x_{1}\left(\lambda y_{1}+y_{1}^{\prime}\right)+x_{1}\left(\lambda y_{2}+y_{2}^{\prime}\right)+x_{2}\left(\lambda y_{1}+y_{1}^{\prime}\right)+3 x_{2}\left(\lambda y_{2}+y_{2}^{\prime}\right) \\
& =\lambda\left(x_{1} y_{1}+x_{1} y_{2}+x_{2} y_{1}+3 x_{2} y_{2}\right)+\left(x_{1} y_{1}^{\prime}+x_{1} y_{2}^{\prime}+x_{2} y_{1}^{\prime}+3 x_{2} y_{2}^{\prime}\right) \\
& =\lambda f(u, v)+f\left(u, v^{\prime}\right) .
\end{aligned}
$$


Similarly, $f\left(\lambda u+u^{\prime}, v\right)=\lambda f(u, v)+f\left(u^{\prime}, v\right)$ for all $u, u^{\prime}, v \in \mathbb{V}$ and $\lambda \in \mathbb{Q}$.

Example 3.3. If $f$ is a rational bilinear form on $\mathbb{V}$, then $f$ is completely determined with the four scalars $a_{i j}=f\left(e_{i}, e_{j}\right)$ by

$$
f(u, v)=\sum_{i, j=1}^{2} a_{i j} x_{i} y_{j} .
$$

If $X$ and $Y$ are the coordinate matrices of $u$ and $v$ in the basis $\mathcal{B}$, and if $A$ is the matrix $2 \times 2$ with entries $a_{i j}=f\left(e_{i}, e_{j}\right)$, then

$$
f(u, v)=X^{t} A Y .
$$

The Example 3.3 can be generalized to describe all the rational bilinear forms on a finite-dimensional vector space $\mathbb{V}$ over the field $\mathbb{Q}$. Let $\mathcal{B}=\left\{e_{1}, \ldots, e_{n}\right\}$ be an ordered basis of $\mathbb{V}$ and $f$ a rational bilinear form on $\mathbb{V}$. If $u=x_{1} e_{1}+\cdots+x_{n} e_{n}, v=y_{1} e_{1}+\cdots+y_{n} e_{n} \in \mathbb{V}$, then

$$
f(u, v)=\sum_{i, j=1}^{n} a_{i j} x_{i} y_{j}=X^{t} A Y,
$$

where $a_{i j}=f\left(e_{i}, e_{j}\right) ; X$ and $Y$ are the coordinate matrices of $u$ and $v$ in the basis $\mathcal{B}$.

Thus, every rational bilinear form on $\mathbb{V}$ is given by

$$
f(u, v)=[u]_{\mathcal{B}}^{t} A[v]_{\mathcal{B}},
$$

for some $n \times n$ matrix $A$ over $\mathbb{Q}$.

Conversely, if $A$ is $n \times n$ matrix over $\mathbb{Q}$, then it is easy to check that (3.4) defines a rational bilinear form on $\mathbb{V}$ such that $a_{i j}=f\left(e_{i}, e_{j}\right)$ (see Example 3.1 and Example 3.2). Then the matrix $A$ is called the matrix of the rational bilinear form $f$ in the basis $\mathcal{B}$, and denoted by $[f]_{\mathcal{B}}$.

Let $L(\mathbb{V}, \mathbb{V}, \mathbb{Q})$ be the vector space of all rational bilinear forms on $\mathbb{V}$.

Theorem 3.1. [6, Theorem X.1] Let $\mathbb{V}$ be a finite-dimensional vector space over the field $\mathbb{Q}$ and $\operatorname{dim} \mathbb{V}=n$. For each ordered basis $\mathcal{B}$ of $\mathbb{V}$, the function that associates with each rational bilinear form on $\mathbb{V}$ its matrix in the ordered basis $\mathcal{B}, f \mapsto[f]_{\mathcal{B}}$, is an isomorphism of the space $L(\mathbb{V}, \mathbb{V}, \mathbb{Q})$ onto the space of $n \times n$ matrices over the field $\mathbb{Q}$.

The formula for the coboundary map is (see $[7,(1.5 .1 .1)]$ )

$$
\begin{aligned}
\beta(f)\left(a_{1}, \ldots, a_{n+1}\right) & =a_{1} f\left(a_{2}, \ldots, a_{n+1}\right) \\
& +\sum_{0<i<n+1}(-1)^{i} f\left(a_{1}, \ldots, a_{i} a_{i+1}, \ldots a_{n+1}\right) \\
& +(-1)^{n+1} f\left(a_{1}, \ldots, a_{n}\right) a_{n+1} .
\end{aligned}
$$

Then $f$ is a 2 -cocycle if $a f(b, c)-f(a b, c)+f(a, b c)-f(a, b) c=0$. If the right action is trivial, $f(a, b) c=$ $f(a, b)$. Writing the left action with exponential notation, $a f(b, c)=f(b, c)^{a}$. Thus, the condition of 2 -cocycle is $f(b, c)^{a}+f(a, b c)=f(a, b)+f(a b, c)$. Using the multiplicative notation for the above condition, it will be said that $f$ satisfies the condition of 2-cocycle if $f(b, c)^{a} f(a, b c)=f(a, b) f(a b, c)$.

Definition 3.2. Let $f$ be a rational bilinear form on $\mathbb{V}, \mathcal{B}$ a basis of $\mathbb{V}$ endowed with structure of group. If exists a left action of $\mathcal{B}$ on $\mathbb{Q}$, then $f$ is a 2 -cocycle on $\mathbb{V}$ if the matrix of $f$ in $\mathcal{B}$ satisfies the condition of $2-$ cocycle and this condition admits multiplicative notation .

Let $\mathcal{B}=G=\langle g\rangle=\{1, g\}$ be the group of automorphisms of the field $\mathbb{Q}(\sqrt{2})$, where $g(1)=1$ and $g(\sqrt{2})=-\sqrt{2}$. Then $\mathbb{V}=\mathbb{Q}[G]$ and $G=G(\mathbb{Q}(\sqrt{2}) / \mathbb{Q})[8$, Example 48.7]. In the case of rational bilinear forms on $\mathbb{V}$, it will use 2 -cocycle to mean 2 -cocycle on $\mathbb{V}$.

Example 3.4. The rational bilinear form on $\mathbb{V}$

$$
f(u, v)=x_{1} y_{1}+x_{1} y_{2}+x_{2} y_{1}+3 x_{2} y_{2}
$$

is a 2 -cocycle.

Since $[f]_{\mathcal{B}}=\left[\begin{array}{ll}f(1,1) & f(1, g) \\ f(g, 1) & f(g, g)\end{array}\right]=\left[\begin{array}{ll}1 & 1 \\ 1 & 3\end{array}\right]$, the condition of 2 -cocycle is satisfied;

i.e., $f(b, c)^{a} f(a, b c)=f(a, b) f(a b, c)$ for all $a, b, c \in \mathcal{B}$. This is expressed with the following eight equalities: 
(1) $1=f(1,1)^{1} f(1,(1)(1))=f(1,1) f((1)(1), 1)$,

(2) $1=f(1, g)^{1} f(1,(1)(g))=f(1,1) f((1)(1), g)$,

(3) $1=f(g, 1)^{1} f(1,(g)(1))=f(1, g) f((1)(g), 1)$,

(4) $3=f(g, g)^{1} f(1,(g)(g))=f(1, g) f((1)(g), g)$,

(5) $1=f(1,1)^{g} f(g,(1)(1))=f(g, 1) f((g)(1), 1)$,

(6) $3=f(1, g)^{g} f(g,(1)(g))=f(g, 1) f((g)(1), g)$,

(7) $3=f(g, 1)^{g} f(g,(g)(1))=f(g, g) f((g)(g), 1)$,

(8) $3=f(g, g)^{g} f\left(g, g^{2}\right)=f(g, g) f\left(g^{2}, g\right)$.

The condition of 2 -cocycle admits multiplicative notation because $f(1,1), f(1, g), f(g, 1)$ and $f(g, g) \in \mathbb{Q}^{*}$.

Example 3.5. The rational bilinear form on $\mathbb{V}$

$$
f(u, v)=2 x_{1} y_{1}+3 x_{2} y_{2}
$$

is not a 2-cocycle.

Since $[f]_{\mathcal{B}}=\left[\begin{array}{ll}f(1,1) & f(1, g) \\ f(g, 1) & f(g, g)\end{array}\right]=\left[\begin{array}{ll}2 & 0 \\ 0 & 3\end{array}\right]$, the condition of 2 -cocycle is not satisfied because

(4) $6=f(g, g)^{1} f(1,(g)(g))=f(1, g) f((1)(g), g)=0$.

Example 3.6. The rational bilinear form on $\mathbb{V}$

$$
f(u, v)=x_{1} y_{1}+x_{1} y_{2}+x_{2} y_{1}
$$

is not a 2 -cocycle.

Since $[f]_{\mathcal{B}}=\left[\begin{array}{ll}f(1,1) & f(1, g) \\ f(g, 1) & f(g, g)\end{array}\right]=\left[\begin{array}{ll}1 & 1 \\ 1 & 0\end{array}\right]$, the condition of 2-cocycle does not admit multiplicative notation because :

(8) $0=f(g, g)^{g} f\left(g, g^{2}\right)=f(g, g) f\left(g^{2}, g\right)$ where $f(g, g)=0$ does not have multiplicative inverse .

Example 3.7. Find all the $2 \times 2$ matrices over $\mathbb{Q}$ which define by (3.4) 2 -cocycles on $\mathbb{V}$.

Let $f$ be a 2 -cocycle on $\mathbb{V}$ such that $[f]_{\mathcal{B}}=\left[\begin{array}{cc}f(1,1) & f(1, g) \\ f(g, 1) & f(g, g)\end{array}\right]=\left[\begin{array}{ll}a_{11} & a_{12} \\ a_{21} & a_{22}\end{array}\right]$. Then the condition of 2-cocycle is satisfied; i.e., $f(b, c)^{a} f(a, b c)=f(a, b) f(a b, c)$ for all $a, b, c \in \mathcal{B}$. This is expressed with the following eight equalities:
(1) $a_{11}{ }^{1} a_{11}=a_{11} a_{11}$,
(5) $a_{11}{ }^{g} a_{21}=a_{21} a_{21}$
(2) $a_{12}{ }^{1} a_{12}=a_{11} a_{12}$
(6) $a_{12}{ }^{g} a_{22}=a_{21} a_{22}$
(3) $a_{21}{ }^{1} a_{12}=a_{12} a_{21}$
(7) $a_{21}{ }^{g} a_{22}=a_{22} a_{11}$
(4) $a_{22}{ }^{1} a_{11}=a_{12} a_{22}$,
(8) $a_{22}{ }^{g} a_{21}=a_{22} a_{12}$

As the condition of 2 -cocycle admits multiplicative notation for $f ; a_{11}, a_{12}, a_{21}$ and $a_{22} \in \mathbb{Q}^{*}$.

Since the action is trivial, from (6) by the cancellation law is obtained that $a_{12}=a_{21}$. Similarly from (7), $a_{21}=a_{11}$. It is noted that $a_{22} \neq 0$ is arbitrary, then setting $a_{11}=a_{12}=a_{21}=a$ and $a_{22}=b$; it follows that $[f]_{\mathcal{B}}=\left[\begin{array}{ll}a & a \\ a & b\end{array}\right]$ where $a$ and $b \in \mathbb{Q}^{*}$.

Thus, in Example $3.4 a=1$ and $b=3$.

Definition 3.3. A 2-cocycle $f$ on $\mathbb{V}$ is said to be normal if $[f]_{\mathcal{B}}=\left[\begin{array}{ll}1 & 1 \\ 1 & b\end{array}\right]$ donde $b \in \mathbb{Q}^{*}$.

If in addition $b=1$ it is said to be the 2 -cocycle $f$ is trivial. In the case in which $b \neq 1, f$ is nontrivial 2-cocycle.

According to Example 3.4, $f(u, v)=x_{1} y_{1}+x_{1} y_{2}+x_{2} y_{1}+3 x_{2} y_{2}$ is nontrivial normal 2-cocycle.

Since $\mathbb{Q}^{*}$ is abelian group, $\mathbb{Q}^{*}$ may be regarded as a trivial left $G$-module for any group $G$ (as in [9, section VI.1]). If $G$ is a finite group, then $\mathbb{V}=\mathbb{Q}[G]$ is finite-dimensional vector space over the field $\mathbb{Q}$, whose basis is $\mathcal{B}=G$. Thus, there exists trivial left action of $\mathcal{B}$ on $\mathbb{Q}$; i.e., $r^{g}=r$ for all $r \in \mathbb{Q}$ and $g \in \mathcal{B}$. One may consider $\mathbb{V}=\mathbb{Q}\left[\mathbb{Z}_{3}\right]$ where $\mathbb{Z}_{3}=\langle g\rangle=\left\{1, g, g^{2}\right\}$. $\mathbb{V}$

Example 3.8. Find all the $3 \times 3$ matrices over $\mathbb{Q}$ which allow to define by (3.4) normal 2 -cocycles on 
Let $f$ be a normal 2-cocycle on $\mathbb{V}$. Proceeding as in the Example 3.7, it results that $[f]_{\mathcal{B}}=\left[\begin{array}{ccc}1 & 1 & 1 \\ 1 & a & b \\ 1 & b & \frac{b}{a}\end{array}\right]$ where $a$ and $b \in \mathbb{Q}^{*}$.

Let $u=x_{1} e_{1}+x_{2} e_{2}+x_{3} e_{3}, v=y_{1} e_{1}+y_{2} e_{2}+y_{3} e_{3}$ be vectors in $\mathbb{V}=\mathbb{Q}\left[\mathbb{Z}_{3}\right]$. Taking $a=1$, the above matrix yields

Example 3.9. The rational bilinear form on $\mathbb{V}$ defined by

$$
f(u, v)=\left[\begin{array}{lll}
x_{1} & x_{2} & x_{3}
\end{array}\right]\left[\begin{array}{lll}
1 & 1 & 1 \\
1 & 1 & b \\
1 & b & b
\end{array}\right]\left[\begin{array}{l}
y_{1} \\
y_{2} \\
y_{3}
\end{array}\right]
$$

is a normal 2-cocycle.

Example 3.10. If one consider the case $\mathbb{V}=\mathbb{Q}\left[\mathbb{Z}_{4}\right]$. Then the matrix

$$
\left(\begin{array}{cccc}
1 & 1 & 1 & 1 \\
1 & a & b & c \\
1 & b & \frac{b c}{a} & \frac{c}{a} \\
1 & c & \frac{c}{a} & \frac{c}{b}
\end{array}\right)
$$

where $a, b$ and $c \in \mathbb{Q}^{*}$; allows to define by (3.4) a normal 2 -cocycle on $\mathbb{V}$.

4. Rational Nontrivial Cocycles for Crossed Products. Let $G$ be a group, $K$ a field and $K^{*}$ the group multiplicative of units of $K$. It is interesting to note the sequence

$$
H\left(G, K^{*}\right) \stackrel{d_{1}}{\longrightarrow} H\left(G \times G, K^{*}\right) \stackrel{d_{2}}{\longrightarrow} H\left(G \times G \times G, K^{*}\right) \stackrel{d_{3}}{\longrightarrow \cdots,}
$$

because $f \in H\left(G \times G, K^{*}\right)$ is a 2 -cocycle if $d_{2}(f)=1$, one can then see that $d_{2}(f): G \times G \times G \rightarrow K^{*}$ is such that

$$
\begin{aligned}
d_{2}(f)(a, b, c) & =\left[[f(b, c)]^{a} f(a, b c)\right]\left[[f(a, b)]_{c} f(a b, c)\right]^{-1}, \text { but right action is trivial } \\
& =\left[[f(b, c)]^{a} f(a, b c)\right][f(a, b) f(a b, c)]^{-1} \\
& =1 .
\end{aligned}
$$

Thus, $f$ is a 2 -cocycle if it satisfies $[f(b, c)]^{a} f(a, b c)=f(a, b) f(a b, c)$.

Denoting $H\left(G \times G, K^{*}\right)$ by $H^{2}\left(G, K^{*}\right)$, the existence of a 2 -cocycle in $H^{2}\left(G(M / K), M^{*}\right)$ compatible with the action is guaranteed by finding $f \in H^{2}\left(G(M / K), M^{*}\right)$ such that $[f(b, c)]^{a} f(a, b c)=f(a, b) f(a b, c)$ for all $a, b, c \in G$. But, for the map $f$ to be 2 -cocycle with respect to a cross product, it is also required that $f$ be normal and that it satisfies the twisted module condition as we will see next.

If $M / K$ is finite and Galois extension of a field $K \& G(M / K)$ is the Galois group of $M$ over $K$, then is possible to find an element $f$ of $H^{2}\left(G(M / K), M^{*}\right)$ such that $M \rtimes_{f} G(M / K)$ is a crossed product. That is, there exists $f \in H^{2}\left(G(M / K), M^{*}\right)$ a normal 2-cocycle compatible with the action $\sigma: G(M / K) \rightarrow$ Aut $(M), g \mapsto \sigma(g): M \rightarrow M$ defined by $\sigma(g)(a)=a^{g}$; such that the twisted module condition holds [3, Corollary 4.6].

4.1. Cocycles for a crossed product $\frac{\mathbb{Q}[X]}{\langle P\rangle} \rtimes_{f} C_{2}$. Let be $M=\mathbb{Q}(\sqrt{2}), K=\mathbb{Q}$ and $G(M / K)$ is the Galois group of $M$ over $K$. Let $P(X)=X^{2}-2$. Since $M=K\left(\Re_{P(X)}\right)$, according to [2, Prop 3.1.5] it is known that $M$ is Galois extension of $\mathbb{Q}$. Since $M=\left\{a_{1}+a_{2} \sqrt{2} \mid a_{1}, a_{2} \in \mathbb{Q}\right\},\{1, \sqrt{2}\}$ is a basis of $M$ over $\mathbb{Q}$. Then $G(M / K)=\{1, g\}$ is described by the table

\begin{tabular}{r|cc} 
& 1 & $g$ \\
\hline 1 & 1 & 1 \\
$\sqrt{2}$ & $\sqrt{2}$ & $-\sqrt{2}$
\end{tabular}

Since $G(M / K)$ is the Galois group of $M$ over $K, G(M / K)$ acts (weakly) on $M$.

Clearly $M=K[\sqrt{2}]=\frac{K[X]}{\langle\operatorname{irr}(\gamma, K)\rangle}$, where $\operatorname{irr}(\gamma, K)=X^{2}-2$, is a field; so the group of units of $M$ is 
$M^{*}=M \backslash\{0\}$.

Let $f: G(M / K) \times G(M / K) \rightarrow M^{*}$ be a function such that $E=M \rtimes_{f} G(M / K)$ is a crossed product. The following three conditions are satisfied according to ([5, page 140] and [3, Corollary 4.6]) :

1) $f\left(1, g^{i}\right)=f\left(g^{i}, 1\right)=1$,

2) $f\left(g^{j}, g^{k}\right)^{g^{i}} f\left(g^{i}, g^{j+k}\right)=f\left(g^{i}, g^{j}\right) f\left(g^{i+j}, g^{k}\right)$,

3) $\left(\left(b_{1}+b_{2} \sqrt{2}\right)^{g^{j}}\right)^{g^{i}} f\left(g^{i}, g^{j}\right)=f\left(g^{i}, g^{j}\right)\left(b_{1}+b_{2} \sqrt{2}\right)^{g^{i+j}}$, where $b_{1}+b_{2} \sqrt{2} \in M$.

All the elements of $G(M / K) \times G(M / K) \times G(M / K)$ are in the following arrangement:

$$
\begin{array}{ll}
(1,1,1) & (1,1, g) \\
(1, g, 1) & (1, g, g) \\
(g, 1,1) & (g, 1, g) \\
(g, g, 1) & (g, g, g)
\end{array}
$$

The assertion 3) for $f,\left(\left(b_{1}+b_{2} \sqrt{2}\right)^{g^{j}}\right)^{g^{i}} f\left(g^{i}, g^{j}\right)=f\left(g^{i}, g^{j}\right)\left(b_{1}+b_{2} \sqrt{2}\right)^{g^{i+j}}$, is satisfied due to that the $K$-algebra $M$ is commutative and $G(M / K) \times M \rightarrow M$, $\left(g^{i}, a_{1}+a_{2} \sqrt{2}\right) \mapsto\left(a_{1}+a_{2} \sqrt{2}\right)^{g^{i}}$ is an action of $G(M / K)$ on $M$.

Considering the above arrangement, the assertion 2) for $f, f\left(g^{j}, g^{k}\right)^{g^{i}} f\left(g^{i}, g^{j+k}\right)=f\left(g^{i}, g^{j}\right) f\left(g^{i+j}, g^{k}\right)$, is expanded to 8 equalities (see Example 3.4).

Since $f\left(g^{j}, g^{k}\right)^{1} f\left(1, g^{j+k}\right)=f\left(1, g^{j}\right) f\left(g^{j}, g^{k}\right) ; f\left(1, g^{k}\right)^{g^{i}} f\left(g^{i}, g^{k}\right)=f\left(g^{i}, 1\right) f\left(g^{i}, g^{k}\right)$ and $f\left(g^{j}, 1\right)^{g^{i}} f\left(g^{i}, g^{j}\right)=f\left(g^{i}, g^{j}\right) f\left(g^{i+j}, 1\right)$; the following equality holds :

(8) $f(g, g)^{g} f\left(g, g^{2}\right)=f(g, g) f\left(g^{2}, g\right)$.

If $f(g, g)=a+b \sqrt{2}, f(g, g)^{g}=a-b \sqrt{2}$. Since $g^{2}=1$, from the equality (8) it is deduced that $f(g, g)=a$, where $a \in \mathbb{Q}^{*}$.

Setting $a=3$ is obtained the matrix $(3)$. Consequently, the rational bilinear form $f$ such that

$$
[f]_{\mathcal{B}}=\left[\begin{array}{ll}
f(1,1) & f(1, g) \\
f(g, 1) & f(g, g)
\end{array}\right]=\left(\begin{array}{ll}
1 & 1 \\
1 & 3
\end{array}\right)
$$

is a nontrivial 2-cocycle such that $A \rtimes_{f} C_{2}$ is a crossed product, where $A=\frac{\mathbb{Q}[X]}{\left\langle X^{2}-2\right\rangle}$ and $C_{2}=\langle g\rangle$ is the Galois group of $X^{2}-2$ over $\mathbb{Q}$.

4.2. Cocycles for a crossed product $\frac{\mathbb{Q}[X]}{\langle P\rangle} \rtimes_{f} C_{3}$. The polynomial $P(X)=X^{3}-7 X+7$ is irreducible in $\mathbb{Q}[X]$ by Eisenstein's criterion with $p=7$. Using the techniques of calculus for polinomial function $P(x)=x^{3}-7 x+7$ as in the proof of [2, Affirmation 2], it is seen that $P(x)$ has three real roots.

Let be $\gamma$ one of the roots of $P=P(X), M=\mathbb{Q}(\gamma), K=\mathbb{Q}$ and $G(M / K)$ is the Galois group of $M$ over $K$. Since $M=K\left(\Re_{P(X)}\right)$, it is known that $M$ is Galois extension of $K$. Since $M=\left\{a_{1}+a_{2} \gamma+\right.$ $\left.a_{3} \gamma^{2} \mid a_{1}, a_{2}, a_{3} \in \mathbb{Q}\right\},\left\{1, \gamma, \gamma^{2}\right\}$ is a basis of $M$ over $\mathbb{Q}$. Then $G(M / K)=\left\{1, g, g^{2}\right\}$ is described by the table

\begin{tabular}{c|ccc} 
& 1 & $g$ & $g^{2}$ \\
\hline 1 & 1 & 1 & 1 \\
$\gamma$ & $\gamma$ & $r_{2}$ & $r_{3}$ \\
$\gamma^{2}$ & $\gamma^{2}$ & $r_{2}^{2}$ & $r_{3}^{2}$
\end{tabular}

where $r_{1}=\gamma, r_{2}=3 \gamma^{2}+4 \gamma-14$ and $r_{3}=-3 \gamma^{2}-5 \gamma+14$ are roots of $\operatorname{irr}(\gamma, K)=X^{3}-7 X+7[?$, page 33].

Since $G(M / K)$ is the Galois group of $M$ over $K, G(M / K)$ acts (weakly) on $M$.

Clearly $M=K(\gamma)=\frac{K[X]}{\langle\operatorname{irr}(\gamma, K)\rangle}$, where $\operatorname{irr}(\gamma, K)=X^{3}-7 X+7$, is a field; so the group of units of $M$ is $M^{*}=M \backslash\{0\}$.

Let $f: G(M / K) \times G(M / K) \rightarrow M^{*}$ be a function such that $E=M \rtimes_{f} G(M / K)$ is a crossed product. Then the following three conditions are satisfied :

1) $f\left(1, g^{i}\right)=f\left(g^{i}, 1\right)=1$, 
2) $f\left(g^{j}, g^{k}\right)^{g^{i}} f\left(g^{i}, g^{j+k}\right)=f\left(g^{i}, g^{j}\right) f\left(g^{i+j}, g^{k}\right)$,

3) $\left(\left(a_{1}+a_{2} \gamma+a_{3} \gamma^{2}\right)^{g^{j}}\right)^{g^{i}} f\left(g^{i}, g^{j}\right)=f\left(g^{i}, g^{j}\right)\left(a_{1}+a_{2} \gamma+a_{3} \gamma^{2}\right)^{g^{i+j}}$,

where $a_{1}+a_{2} \gamma+a_{3} \gamma^{2} \in M$.

All the elements of $G(M / K) \times G(M / K) \times G(M / K)$ are in the following arrangement:

$\begin{array}{llllllll}(1,1,1) & (1,1, g) & \left(1,1, g^{2}\right) & (1, g, 1) & (1, g, g) & \left(1, g, g^{2}\right) & \left(1, g^{2}, 1\right) & \left(1, g^{2}, g\right) \\ \left(1, g^{2}, g^{2}\right) & (g, 1,1) & (g, 1, g) & \left(g, 1, g^{2}\right) & (g, g, 1) & (g, g, g) & \left(g, g, g^{2}\right) & \left(g, g^{2}, 1\right) \\ \left(g, g^{2}, g\right) & \left(g, g^{2}, g^{2}\right) & \left(g^{2}, 1,1\right) & \left(g^{2}, 1, g\right) & \left(g^{2}, 1, g^{2}\right) & \left(g^{2}, g, 1\right) & \left(g^{2}, g, g\right) & \left(g^{2}, g, g^{2}\right) \\ \left(g^{2}, g^{2}, 1\right) & \left(g^{2}, g^{2}, g\right) & \left(g^{2}, g^{2}, g^{2}\right) & & & & & \end{array}$

Considering this arrangement, the assertion 2) for $f, f\left(g^{j}, g^{k}\right)^{g^{i}} f\left(g^{i}, g^{j+k}\right)=f\left(g^{i}, g^{j}\right) f\left(g^{i+j}, g^{k}\right)$, is expanded to 27 equalities.

Since $f\left(g^{j}, g^{k}\right)^{1} f\left(1, g^{j+k}\right)=f\left(1, g^{j}\right) f\left(g^{j}, g^{k}\right) ; f\left(1, g^{k}\right)^{g^{i}} f\left(g^{i}, g^{k}\right)=f\left(g^{i}, 1\right) f\left(g^{i}, g^{k}\right)$ and $f\left(g^{j}, 1\right)^{g^{i}} f\left(g^{i}, g^{j}\right)=f\left(g^{i}, g^{j}\right) f\left(g^{i+j}, 1\right)$; the following 8 equalities hold :

(14) $f(g, g)^{g} f\left(g, g^{2}\right)=f(g, g) f\left(g^{2}, g\right)$,

(15) $f\left(g, g^{2}\right)^{g}=f(g, g) f\left(g^{2}, g^{2}\right)$,

(17) $f\left(g^{2}, g\right)^{g}=f\left(g, g^{2}\right)$,

(18) $f\left(g^{2}, g^{2}\right)^{g} f(g, g)=f\left(g, g^{2}\right)$,

(23) $f(g, g)^{g^{2}} f\left(g^{2}, g^{2}\right)=f\left(g^{2}, g\right)$

(24) $f\left(g, g^{2}\right)^{g^{2}}=f\left(g^{2}, g\right)$,

(26) $f\left(g^{2}, g\right)^{g^{2}}=f\left(g^{2}, g^{2}\right) f(g, g)$,

(27) $f\left(g^{2}, g^{2}\right)^{g^{2}} f\left(g^{2}, g\right)=f\left(g^{2}, g^{2}\right) f\left(g, g^{2}\right)$.

If $a, b \in \mathbb{Q}^{*}$, then the matrix

$$
\left(\begin{array}{cc}
f(g, g) & f\left(g, g^{2}\right) \\
f\left(g^{2}, g\right) & f\left(g^{2}, g^{2}\right)
\end{array}\right)=\left(\begin{array}{cc}
a & b \\
b & \frac{b}{a}
\end{array}\right)
$$

presents a solution of the above system of 8 equations.

Therefore, the rational bilinear form $f$ of Example 3.8 given by

$$
[f]_{\mathcal{B}}=\left[\begin{array}{ccc}
1 & 1 & 1 \\
1 & a & b \\
1 & b & \frac{b}{a}
\end{array}\right]
$$

is a nontrivial 2-cocycle such that $A \rtimes_{f} C_{3}$ is a crossed product, where $A=\frac{\mathbb{Q}[X]}{\left\langle X^{3}-7 X+7\right\rangle}$ and $C_{3}=\langle g\rangle$ is the Galois group of $X^{3}-7 X+7$ over $\mathbb{Q}$.

4.3. Cocycles for a crossed product $\frac{\mathbb{Q}[X]}{\langle P\rangle} \rtimes_{f} G_{4}$. Let be $M=\mathbb{Q}(\sqrt{2}, \sqrt{3}), K=\mathbb{Q}$ and $G(M / K)$ is the Galois group of $M$ over $K$. According to Fraleigh [8, Example 53.3] it is known that $M$ is Galois extension of $\mathbb{Q}$.

Since $M=\left\{a_{1}+a_{2} \sqrt{2}+a_{3} \sqrt{3}+a_{4} \sqrt{6} \mid a_{1}, a_{2}, a_{3}, a_{4} \in \mathbb{Q}\right\}$, then $G(M / K)=\left\{g_{0}, g_{1}, g_{2}, g_{3}\right\}$ is described by

\begin{tabular}{r|cccc} 
& $g_{0}$ & $g_{1}$ & $g_{2}$ & $g_{3}$ \\
\hline 1 & 1 & 1 & 1 & 1 \\
$\sqrt{2}$ & $\sqrt{2}$ & $-\sqrt{2}$ & $\sqrt{2}$ & $-\sqrt{2}$ \\
$\sqrt{3}$ & $\sqrt{3}$ & $\sqrt{3}$ & $-\sqrt{3}$ & $-\sqrt{3}$ \\
$\sqrt{6}$ & $\sqrt{6}$ & $-\sqrt{6}$ & $-\sqrt{6}$ & $\sqrt{6}$
\end{tabular}


Hence, the group table for $G(M / K)$ is given by

\begin{tabular}{l|llll} 
& $g_{0}$ & $g_{1}$ & $g_{2}$ & $g_{3}$ \\
\hline$g_{0}$ & $g_{0}$ & $g_{1}$ & $g_{2}$ & $g_{3}$ \\
$g_{1}$ & $g_{1}$ & $g_{0}$ & $g_{3}$ & $g_{2}$ \\
$g_{2}$ & $g_{2}$ & $g_{3}$ & $g_{0}$ & $g_{1}$ \\
$g_{3}$ & $g_{3}$ & $g_{2}$ & $g_{1}$ & $g_{0}$.
\end{tabular}

It is considered $\sigma: G(M / K) \rightarrow$ Aut $(M), g_{i} \mapsto \sigma\left(g_{i}\right): M \rightarrow M$ defined by $\sigma\left(g_{i}\right)\left(a_{1}+a_{2} \sqrt{2}+a_{3} \sqrt{3}+a_{4} \sqrt{6}\right)=\left(a_{1}+a_{2} \sqrt{2}+a_{3} \sqrt{3}+a_{4} \sqrt{6}\right)^{g_{i}}$.

Recalls that $G(M / K) \times G(M / K)=\left\{\left(g_{0}, g_{0}\right),\left(g_{0}, g_{1}\right),\left(g_{0}, g_{2}\right),\left(g_{0}, g_{3}\right),\left(g_{1}, g_{0}\right),\left(g_{1}, g_{1}\right)\right.$, $\left.\left(g_{1}, g_{2}\right),\left(g_{1}, g_{3}\right),\left(g_{2}, g_{0}\right),\left(g_{2}, g_{1}\right),\left(g_{2}, g_{2}\right),\left(g_{2}, g_{3}\right),\left(g_{3}, g_{0}\right),\left(g_{3}, g_{1}\right),\left(g_{3}, g_{2}\right),\left(g_{3}, g_{3}\right)\right\}$.

If $\left(g_{i}, g_{j}\right) \in G(M / K) \times G(M / K)$, then $\sigma\left(g_{i} g_{j}\right)=\sigma\left(g_{i}\right) \sigma\left(g_{j}\right)$.

In fact, if $a_{1}+a_{2} \sqrt{2}+a_{3} \sqrt{3}+a_{4} \sqrt{6} \in M$, then by (4.3) \& (4.4):

$$
\begin{aligned}
\sigma\left(g_{1} g_{3}\right)\left(a_{1}+a_{2} \sqrt{2}+a_{3} \sqrt{3}+a_{4} \sqrt{6}\right) & =\sigma\left(g_{2}\right)\left(a_{1}+a_{2} \sqrt{2}+a_{3} \sqrt{3}+a_{4} \sqrt{6}\right) \\
& =a_{1}+a_{2} \sqrt{2}-a_{3} \sqrt{3}-a_{4} \sqrt{6}, \\
\sigma\left(g_{1}\right) \sigma\left(g_{3}\right)\left(a_{1}+a_{2} \sqrt{2}+a_{3} \sqrt{3}+a_{4} \sqrt{6}\right) & =\sigma\left(g_{1}\right)\left(a_{1}-a_{2} \sqrt{2}-a_{3} \sqrt{3}+a_{4} \sqrt{6}\right) \\
& =a_{1}+a_{2} \sqrt{2}-a_{3} \sqrt{3}-a_{4} \sqrt{6} .
\end{aligned}
$$

The others equalities are checked similarly.

Since $G(M / K)$ is the Galois group of $M$ over $K$, the map $\sigma\left(g_{i}\right)$ is a $K$-algebra automorphism of $M$. That is; if $\lambda \in K, a_{1}+a_{2} \sqrt{2}+a_{3} \sqrt{3}+a_{4} \sqrt{6}$ and $b_{1}+b_{2} \sqrt{2}+b_{3} \sqrt{3}+b_{4} \sqrt{6} \in M$, then :

$$
\begin{aligned}
\text { 1) } \sigma\left(g_{i}\right)\left(\lambda\left(a_{1}+a_{2} \sqrt{2}+a_{3} \sqrt{3}+a_{4} \sqrt{6}\right)\right)=\lambda \sigma\left(g_{i}\right)\left(a_{1}+a_{2} \sqrt{2}+a_{3} \sqrt{3}+a_{4} \sqrt{6}\right), \\
\text { 2) } \sigma\left(g_{i}\right)\left(\left(a_{1}+a_{2} \sqrt{2}+a_{3} \sqrt{3}+a_{4} \sqrt{6}\right)+\left(b_{1}+b_{2} \sqrt{2}+b_{3} \sqrt{3}+b_{4} \sqrt{6}\right)\right) \\
=\sigma\left(g_{i}\right)\left(a_{1}+a_{2} \sqrt{2}+a_{3} \sqrt{3}+a_{4} \sqrt{6}\right)+\sigma\left(g_{i}\right)\left(b_{1}+b_{2} \sqrt{2}+b_{3} \sqrt{3}+b_{4} \sqrt{6}\right), \\
3) \sigma\left(g_{i}\right)\left(\left(a_{1}+a_{2} \sqrt{2}+a_{3} \sqrt{3}+a_{4} \sqrt{6}\right)\left(b_{1}+b_{2} \sqrt{2}+b_{3} \sqrt{3}+b_{4} \sqrt{6}\right)\right) \\
=\sigma\left(g_{i}\right)\left(a_{1}+a_{2} \sqrt{2}+a_{3} \sqrt{3}+a_{4} \sqrt{6}\right) \sigma\left(g_{i}\right)\left(b_{1}+b_{2} \sqrt{2}+b_{3} \sqrt{3}+b_{4} \sqrt{6}\right) .
\end{aligned}
$$

Therefore, $G(M / K)$ acts (weakly) on $M$.

By the primitive element theorem [2, p 74] exists $\gamma \in M$ such that $M=K[\gamma]=\frac{K[X]}{\langle\operatorname{irr}(\gamma, K)\rangle}$ is a field; so the group of units of $M$ is $M^{*}=M \backslash\{0\}$.

For example $(1+\sqrt{2}+\sqrt{3}+\sqrt{6})^{-1}=\frac{1}{2}(1-\sqrt{2}-\sqrt{3}+\sqrt{6})$.

If $\gamma=1+\sqrt{2}+\sqrt{3}+\sqrt{6}$, then $\operatorname{irr}(\gamma, K)=X^{4}-4 X^{3}-10 X^{2}-20 X-23$.

Let $f: G(M / K) \times G(M / K) \rightarrow M^{*}$ be a function such that $E=M \rtimes_{f} G(M / K)$ is a crossed product. Then the following three conditions are satisfied :

1) $f\left(g_{0}, g_{i}\right)=f\left(g_{i}, g_{0}\right)=1$,

2) $f\left(g_{j}, g_{k}\right)^{g_{i}} f\left(g_{i}, g_{j} g_{k}\right)=f\left(g_{i}, g_{j}\right) f\left(g_{i} g_{j}, g_{k}\right)$,

3) $\left(\left(b_{1}+b_{2} \sqrt{2}+b_{3} \sqrt{3}+b_{4} \sqrt{6}\right)^{g_{j}}\right)^{g_{i}} f\left(g_{i}, g_{j}\right)=f\left(g_{i}, g_{j}\right)\left(b_{1}+b_{2} \sqrt{2}+b_{3} \sqrt{3}+b_{4} \sqrt{6}\right)^{g_{i} g_{j}}$, where $b_{1}+b_{2} \sqrt{2}+b_{3} \sqrt{3}+b_{4} \sqrt{6} \in M$.

All the elements of $G(M / K) \times G(M / \dot{K}) \times G(M / K)$ are the following:

$\begin{array}{llllllll}\left(g_{0}, g_{0}, g_{0}\right) & \left(g_{0}, g_{0}, g_{1}\right) & \left(g_{0}, g_{0}, g_{2}\right) & \left(g_{0}, g_{0}, g_{3}\right) & \left(g_{0}, g_{1}, g_{0}\right) & \left(g_{0}, g_{1}, g_{1}\right) & \left(g_{0}, g_{1}, g_{2}\right) & \left(g_{0}, g_{1}, g_{3}\right) \\ \left(g_{0}, g_{2}, g_{0}\right) & \left(g_{0}, g_{2}, g_{1}\right) & \left(g_{0}, g_{2}, g_{2}\right) & \left(g_{0}, g_{2}, g_{3}\right) & \left(g_{0}, g_{3}, g_{0}\right) & \left(g_{0}, g_{3}, g_{1}\right) & \left(g_{0}, g_{3}, g_{2}\right) & \left(g_{0}, g_{3}, g_{3}\right) \\ \left(g_{1}, g_{0}, g_{0}\right) & \left(g_{1}, g_{0}, g_{1}\right) & \left(g_{1}, g_{0}, g_{2}\right) & \left(g_{1}, g_{0}, g_{3}\right) & \left(g_{1}, g_{1}, g_{0}\right) & \left(g_{1}, g_{1}, g_{1}\right) & \left(g_{1}, g_{1}, g_{2}\right) & \left(g_{1}, g_{1}, g_{3}\right) \\ \left(g_{1}, g_{2}, g_{0}\right) & \left(g_{1}, g_{2}, g_{1}\right) & \left(g_{1}, g_{2}, g_{2}\right) & \left(g_{1}, g_{2}, g_{3}\right) & \left(g_{1}, g_{3}, g_{0}\right) & \left(g_{1}, g_{3}, g_{1}\right) & \left(g_{1}, g_{3}, g_{2}\right) & \left(g_{1}, g_{3}, g_{3}\right) \\ \left(g_{2}, g_{0}, g_{0}\right) & \left(g_{2}, g_{0}, g_{1}\right) & \left(g_{2}, g_{0}, g_{2}\right) & \left(g_{2}, g_{0}, g_{3}\right) & \left(g_{2}, g_{1}, g_{0}\right) & \left(g_{2}, g_{1}, g_{1}\right) & \left(g_{2}, g_{1}, g_{2}\right) & \left(g_{2}, g_{1}, g_{3}\right) \\ \left(g_{2}, g_{2}, g_{0}\right) & \left(g_{2}, g_{2}, g_{1}\right) & \left(g_{2}, g_{2}, g_{2}\right) & \left(g_{2}, g_{2}, g_{3}\right) & \left(g_{2}, g_{3}, g_{0}\right) & \left(g_{2}, g_{3}, g_{1}\right) & \left(g_{2}, g_{3}, g_{2}\right) & \left(g_{2}, g_{3}, g_{3}\right) \\ \left(g_{3}, g_{0}, g_{0}\right) & \left(g_{3}, g_{0}, g_{1}\right) & \left(g_{3}, g_{0}, g_{2}\right) & \left(g_{3}, g_{0}, g_{3}\right) & \left(g_{3}, g_{1}, g_{0}\right) & \left(g_{3}, g_{1}, g_{1}\right) & \left(g_{3}, g_{1}, g_{2}\right) & \left(g_{3}, g_{1}, g_{3}\right) \\ \left(g_{3}, g_{2}, g_{0}\right) & \left(g_{3}, g_{2}, g_{1}\right) & \left(g_{3}, g_{2}, g_{2}\right) & \left(g_{3}, g_{2}, g_{3}\right) & \left(g_{3}, g_{3}, g_{0}\right) & \left(g_{3}, g_{3}, g_{1}\right) & \left(g_{3}, g_{3}, g_{2}\right) & \left(g_{3}, g_{3}, g_{3}\right)\end{array}$


Using the group table (4.4), $f\left(g_{j}, g_{k}\right)^{g_{i}} f\left(g_{i}, g_{j} g_{k}\right)=f\left(g_{i}, g_{j}\right) f\left(g_{i} g_{j}, g_{k}\right)$ is expanded to 64 equalities. Since $f\left(g_{j}, g_{k}\right)^{g_{0}} f\left(g_{0}, g_{j} g_{k}\right)=f\left(g_{0}, g_{j}\right) f\left(g_{j}, g_{k}\right) ; f\left(g_{0}, g_{k}\right)^{g_{i}} f\left(g_{i}, g_{k}\right)=f\left(g_{i}, g_{0}\right) f\left(g_{i}, g_{k}\right)$ and $f\left(g_{j}, g_{0}\right)^{g_{i}} f\left(g_{i}, g_{j}\right)=f\left(g_{i}, g_{j}\right) f\left(g_{i} g_{j}, g_{0}\right)$; the following 27 equalities hold :

(22) $f\left(g_{1}, g_{1}\right)^{g_{1}}=f\left(g_{1}, g_{1}\right)$,

(23) $f\left(g_{1}, g_{2}\right)^{g_{1}} f\left(g_{1}, g_{3}\right)=f\left(g_{1}, g_{1}\right)$,

(24) $f\left(g_{1}, g_{3}\right)^{g_{1}} f\left(g_{1}, g_{2}\right)=f\left(g_{1}, g_{1}\right)$,

(26) $f\left(g_{2}, g_{1}\right)^{g_{1}} f\left(g_{1}, g_{3}\right)=f\left(g_{1}, g_{2}\right) f\left(g_{3}, g_{1}\right)$,

(27) $f\left(g_{2}, g_{2}\right)^{g_{1}}=f\left(g_{1}, g_{2}\right) f\left(g_{3}, g_{2}\right)$,

(28) $f\left(g_{2}, g_{3}\right)^{g_{1}} f\left(g_{1}, g_{1}\right)=f\left(g_{1}, g_{2}\right) f\left(g_{3}, g_{3}\right)$,

(30) $f\left(g_{3}, g_{1}\right)^{g_{1}} f\left(g_{1}, g_{2}\right)=f\left(g_{1}, g_{3}\right) f\left(g_{2}, g_{1}\right)$,

(31) $f\left(g_{3}, g_{2}\right)^{g_{1}} f\left(g_{1}, g_{1}\right)=f\left(g_{1}, g_{3}\right) f\left(g_{2}, g_{2}\right)$,

(32) $f\left(g_{3}, g_{3}\right)^{g_{1}}=f\left(g_{1}, g_{3}\right) f\left(g_{2}, g_{3}\right)$,

(38) $f\left(g_{1}, g_{1}\right)^{g_{2}}=f\left(g_{2}, g_{1}\right) f\left(g_{3}, g_{1}\right)$,

(39) $f\left(g_{1}, g_{2}\right)^{g_{2}} f\left(g_{2}, g_{3}\right)=f\left(g_{2}, g_{1}\right) f\left(g_{3}, g_{2}\right)$,

(40) $f\left(g_{1}, g_{3}\right)^{g_{2}} f\left(g_{2}, g_{2}\right)=f\left(g_{2}, g_{1}\right) f\left(g_{3}, g_{3}\right)$,

(42) $f\left(g_{2}, g_{1}\right)^{g_{2}} f\left(g_{2}, g_{3}\right)=f\left(g_{2}, g_{2}\right)$,

(43) $f\left(g_{2}, g_{2}\right)^{g_{2}}=f\left(g_{2}, g_{2}\right)$,
(44) $f\left(g_{2}, g_{3}\right)^{g_{2}} f\left(g_{2}, g_{1}\right)=f\left(g_{2}, g_{2}\right)$,

(46) $f\left(g_{3}, g_{1}\right)^{g_{2}} f\left(g_{2}, g_{2}\right)=f\left(g_{2}, g_{3}\right) f\left(g_{1}, g_{1}\right)$,

(47) $f\left(g_{3}, g_{2}\right)^{g_{2}} f\left(g_{2}, g_{1}\right)=f\left(g_{2}, g_{3}\right) f\left(g_{1}, g_{2}\right)$,

(48) $f\left(g_{3}, g_{3}\right)^{g_{2}}=f\left(g_{2}, g_{3}\right) f\left(g_{1}, g_{3}\right)$,

(54) $f\left(g_{1}, g_{1}\right)^{g_{3}}=f\left(g_{3}, g_{1}\right) f\left(g_{2}, g_{1}\right)$,

(55) $f\left(g_{1}, g_{2}\right)^{g_{3}} f\left(g_{3}, g_{3}\right)=f\left(g_{3}, g_{1}\right) f\left(g_{2}, g_{2}\right)$,

(56) $f\left(g_{1}, g_{3}\right)^{g_{3}} f\left(g_{3}, g_{2}\right)=f\left(g_{3}, g_{1}\right) f\left(g_{2}, g_{3}\right)$,

(58) $f\left(g_{2}, g_{1}\right)^{g_{3}} f\left(g_{3}, g_{3}\right)=f\left(g_{3}, g_{2}\right) f\left(g_{1}, g_{1}\right)$,

(59) $f\left(g_{2}, g_{2}\right)^{g_{3}}=f\left(g_{3}, g_{2}\right) f\left(g_{1}, g_{2}\right)$,

(60) $f\left(g_{2}, g_{3}\right)^{g_{3}} f\left(g_{3}, g_{1}\right)=f\left(g_{3}, g_{2}\right) f\left(g_{1}, g_{3}\right)$,

(62) $f\left(g_{3}, g_{1}\right)^{g_{3}} f\left(g_{3}, g_{2}\right)=f\left(g_{3}, g_{3}\right)$,

(63) $f\left(g_{3}, g_{2}\right)^{g_{3}} f\left(g_{3}, g_{1}\right)=f\left(g_{3}, g_{3}\right)$,

(64) $f\left(g_{3}, g_{3}\right)^{g_{3}}=f\left(g_{3}, g_{3}\right)$.

If $f\left(g_{i}, g_{j}\right)=a_{i j}+b_{i j} \sqrt{2}+c_{i j} \sqrt{3}+d_{i j} \sqrt{6}, f\left(g_{1}, g_{1}\right)^{g_{1}}=a_{11}-b_{11} \sqrt{2}+c_{11} \sqrt{3}-d_{11} \sqrt{6}$. From $f\left(g_{1}, g_{1}\right)^{g_{1}}=f\left(g_{1}, g_{1}\right)$, it follows that $f\left(g_{1}, g_{1}\right)=a_{11}+c_{11} \sqrt{3}$. Similarly from $f\left(g_{2}, g_{2}\right)^{g_{2}}=f\left(g_{2}, g_{2}\right)$ and $f\left(g_{3}, g_{3}\right)^{g_{3}}=f\left(g_{3}, g_{3}\right)$ it is obtained $f\left(g_{2}, g_{2}\right)=a_{22}+b_{22} \sqrt{2}, f\left(g_{3}, g_{3}\right)=a_{33}+d_{33} \sqrt{6}$ respectively. We note that the equations (27) with (59), (32) with (48) \& (38) with (54) are the same. Then we must resolve a system of 24 equations.

By the condition of normality 1$)$ of $f$, exist 9 variables $f\left(g_{i}, g_{j}\right)$ for $i, j: 1,2,3$. Furthermore, each of these variables depends on 4 variables. Thus, exist 36 variables. That is, one has a system of 24 equations in 36 variables.

Since $f\left(g_{1}, g_{2}\right)^{g_{1}}=a_{12}-b_{12} \sqrt{2}+c_{12} \sqrt{3}-d_{12} \sqrt{6} ; f\left(g_{1}, g_{3}\right)=a_{13}+b_{13} \sqrt{2}+c_{13} \sqrt{3}+d_{13} \sqrt{6}$, and $(a+b \sqrt{2}+c \sqrt{3}+d \sqrt{6})\left(a^{\prime}+b^{\prime} \sqrt{2}+c^{\prime} \sqrt{3}+d^{\prime} \sqrt{6}\right)=\left(a a^{\prime}+2 b b^{\prime}+3 c c^{\prime}+6 d d^{\prime}\right)+\left(a b^{\prime}+b a^{\prime}+3 c d^{\prime}+3 d c^{\prime}\right) \sqrt{2}+$ $\left(a c^{\prime}+2 b d^{\prime}+c a^{\prime}+2 d b^{\prime}\right) \sqrt{3}+\left(a d^{\prime}+b c^{\prime}+c b^{\prime}+d a^{\prime}\right) \sqrt{6}$, the equation $f\left(g_{1}, g_{2}\right)^{g_{1}} f\left(g_{1}, g_{3}\right)=f\left(g_{1}, g_{1}\right)$ is written as

$$
\left\{\begin{array}{l}
a_{12} a_{13}-2 b_{12} b_{13}+3 c_{12} c_{13}-6 d_{12} d_{13}=a_{11} \\
a_{12} b_{13}-b_{12} a_{13}+3 c_{12} d_{13}-3 d_{12} c_{13}=0 \\
a_{12} c_{13}-2 b_{12} d_{13}+c_{12} a_{13}-2 d_{12} b_{13}=c_{11} \\
a_{12} d_{13}-b_{12} c_{13}+c_{12} b_{13}-d_{12} a_{13}=0 .
\end{array}\right.
$$

Since $f\left(g_{1}, g_{3}\right)^{g_{1}}=a_{13}-b_{13} \sqrt{2}+c_{13} \sqrt{3}-d_{13} \sqrt{6}$ and $f\left(g_{1}, g_{2}\right)=a_{12}+b_{12} \sqrt{2}+c_{12} \sqrt{3}+d_{12} \sqrt{6}$, the equation $f\left(g_{1}, g_{3}\right)^{g_{1}} f\left(g_{1}, g_{2}\right)=f\left(g_{1}, g_{1}\right)$ is written as

$$
\left\{\begin{array}{l}
a_{13} a_{12}-2 b_{13} b_{12}+3 c_{13} c_{12}-6 d_{13} d_{12}=a_{11} \\
a_{13} b_{12}-b_{13} a_{12}+3 c_{13} d_{12}-3 d_{13} c_{12}=0 \\
a_{13} c_{12}-2 b_{13} d_{12}+c_{13} a_{12}-2 d_{13} b_{12}=c_{11} \\
a_{13} d_{12}-b_{13} c_{12}+c_{13} b_{12}-d_{13} a_{12}=0 .
\end{array}\right.
$$

Therefore the equations (23) with (24) are the same. Similarly, the equations (42) with (44) \& (62) with (63) are the same. So, the system is reduced to one of 21 equations in 36 variables.

If $a_{11}, a_{13}$ and $a_{22} \in \mathbb{Q}^{*}$, then the matrix

$$
\left(\begin{array}{lll}
f\left(g_{1}, g_{1}\right) & f\left(g_{1}, g_{2}\right) & f\left(g_{1}, g_{3}\right) \\
f\left(g_{2}, g_{1}\right) & f\left(g_{2}, g_{2}\right) & f\left(g_{2}, g_{3}\right) \\
f\left(g_{3}, g_{1}\right) & f\left(g_{3}, g_{2}\right) & f\left(g_{3}, g_{3}\right)
\end{array}\right)
$$


can be written as

$$
\left(\begin{array}{ccc}
a_{11} & \frac{a_{11}}{a_{13}} & a_{13} \\
\frac{a_{11}}{a_{13}} & a_{22} & \frac{a_{13} a_{22}}{a_{11}} \\
a_{13} & \frac{a_{13} a_{22}}{a_{11}} & \frac{a_{13}^{2} a_{22}}{a_{11}}
\end{array}\right) \text { or }\left(\begin{array}{ccc}
a_{11} & \frac{a_{11}}{a_{13}} & a_{13} \\
-\frac{a_{11}}{a_{13}} & a_{22} & -\frac{a_{13} a_{22}}{a_{11}} \\
-a_{13} & \frac{a_{13} a_{22}}{a_{11}} & -\frac{a_{13}^{2} a_{22}}{a_{11}}
\end{array}\right) \text {. }
$$

This matrix presents a solution of the above system of 21 equations with 36 variables. If $a_{11}=a_{13}=1 \& a_{22}=4$, it is obtained the matrix

$$
\left(\begin{array}{ccc}
1 & 1 & 1 \\
-1 & 4 & -4 \\
-1 & 4 & -4
\end{array}\right)
$$

Consequently, the rational bilinear form $f$ such that

$$
[f]_{\mathcal{B}}=\left(\begin{array}{cccc}
1 & 1 & 1 & 1 \\
1 & 1 & 1 & 1 \\
1 & -1 & 4 & -4 \\
1 & -1 & 4 & -4
\end{array}\right)
$$

is a nontrivial $2-$ cocycle such that $\frac{\mathbb{Q}[X]}{\langle P\rangle} \rtimes_{f} G_{4}$ is a crossed product, where $P=X^{4}-5 X^{2}+6$ and $G_{4}$ is the Galois group of $P$ over $\mathbb{Q}$.

4.3.1. Computing Nonrational 2 -cocycles. From $(22) f\left(g_{1}, g_{1}\right)^{g_{1}}=f\left(g_{1}, g_{1}\right)$, one has $f\left(g_{1}, g_{1}\right)=a_{11}+c_{11} \sqrt{3}$. If $f\left(g_{1}, g_{3}\right)=b_{13} \sqrt{2}$ where $b_{13} \in \mathbb{Q}^{*}$, then $a_{13}=c_{13}=d_{13}=0$.

$$
\text { Since } \begin{aligned}
f\left(g_{1}, g_{2}\right)^{g_{1}} f\left(g_{1}, g_{3}\right) & =\left(a_{12}-b_{12} \sqrt{2}+c_{12} \sqrt{3}-d_{12} \sqrt{6}\right) b_{13} \sqrt{2} \\
& =-2 b_{12} b_{13}+a_{12} b_{13} \sqrt{2}-2 d_{12} b_{13} \sqrt{3}+c_{12} b_{13} \sqrt{6}
\end{aligned}
$$

$f\left(g_{1}, g_{1}\right)=a_{11}+c_{11} \sqrt{3}$, from $(23) f\left(g_{1}, g_{2}\right)^{g_{1}} f\left(g_{1}, g_{3}\right)=f\left(g_{1}, g_{1}\right)$ one obtains $-2 b_{12} b_{13}=a_{11}, a_{12}=0,-2 d_{12} b_{13}=c_{11}$ and $c_{12}=0$. If $c_{11}=0$, then $f\left(g_{1}, g_{1}\right)=a_{11}$ and $f\left(g_{1}, g_{2}\right)=-\frac{a_{11}}{2 b_{13}} \sqrt{2}$.

$$
\text { Since } \begin{aligned}
f\left(g_{2}, g_{1}\right)^{g_{1}} f\left(g_{1}, g_{3}\right) & =\left(a_{21}-b_{21} \sqrt{2}+c_{21} \sqrt{3}-d_{21} \sqrt{6}\right) b_{13} \sqrt{2} \\
& =-2 b_{21} b_{13}+a_{21} b_{13} \sqrt{2}-2 d_{21} b_{13} \sqrt{3}+c_{21} b_{13} \sqrt{6} ; \\
f\left(g_{1}, g_{2}\right) f\left(g_{3}, g_{1}\right) & =-\frac{a_{11}}{2 b_{13}} \sqrt{2}\left(a_{31}+b_{31} \sqrt{2}+c_{31} \sqrt{3}+d_{31} \sqrt{6}\right) \\
& =-\frac{a_{11} b_{31}}{b_{13}}-\frac{a_{11} a_{31}}{2 b_{13}} \sqrt{2}-\frac{a_{11} d_{31}}{b_{13}} \sqrt{3}-\frac{a_{11} c_{31}}{2 b_{13}} \sqrt{6}
\end{aligned}
$$

from $(26) f\left(g_{2}, g_{1}\right)^{g_{1}} f\left(g_{1}, g_{3}\right)=f\left(g_{1}, g_{2}\right) f\left(g_{3}, g_{1}\right)$ it follows that

$$
2 b_{21} b_{13}=\frac{a_{11} b_{31}}{b_{13}}, a_{21} b_{13}=-\frac{a_{11} a_{31}}{2 b_{13}}, 2 d_{21} b_{13}=\frac{a_{11} d_{31}}{b_{13}} \text { and } c_{21} b_{13}=-\frac{a_{11} c_{31}}{2 b_{13}} \text {. }
$$

From $(43) f\left(g_{2}, g_{2}\right)^{g_{2}}=f\left(g_{2}, g_{2}\right)$, one obtains $f\left(g_{2}, g_{2}\right)=a_{22}+b_{22} \sqrt{2}$. If $b_{22}=0, f\left(g_{2}, g_{2}\right)=a_{22}$.

$$
\text { Since } \begin{aligned}
f\left(g_{1}, g_{2}\right) f\left(g_{3}, g_{2}\right) & =-\frac{a_{11}}{2 b_{13}} \sqrt{2}\left(a_{32}+b_{32} \sqrt{2}+c_{32} \sqrt{3}+d_{32} \sqrt{6}\right) \\
& =-\frac{a_{11} b_{32}}{b_{13}}-\frac{a_{11} a_{32}}{2 b_{13}} \sqrt{2}-\frac{a_{11} d_{32}}{b_{13}} \sqrt{3}-\frac{a_{11} c_{32}}{2 b_{13}} \sqrt{6}
\end{aligned}
$$

from $(27) f\left(g_{2}, g_{2}\right)^{g_{1}}=f\left(g_{1}, g_{2}\right) f\left(g_{3}, g_{2}\right)$ one obtains that $b_{32}=-\frac{a_{22} b_{13}}{a_{11}}$,

$a_{32}=c_{32}=d_{32}=0$. Thus $f\left(g_{3}, g_{2}\right)=-\frac{a_{22} b_{13}}{a_{11}} \sqrt{2}$. 
From $(64) f\left(g_{3}, g_{3}\right)^{g_{3}}=f\left(g_{3}, g_{3}\right)$, one has $f\left(g_{3}, g_{3}\right)=a_{33}+d_{33} \sqrt{6}$.

$$
\text { Since } \begin{aligned}
f\left(g_{2}, g_{3}\right)^{g_{1}} f\left(g_{1}, g_{1}\right) & =\left(a_{23}-b_{23} \sqrt{2}+c_{23} \sqrt{3}-d_{23} \sqrt{6}\right) a_{11} \\
& =a_{23} a_{11}-b_{23} a_{11} \sqrt{2}+c_{23} a_{11} \sqrt{3}-d_{23} a_{11} \sqrt{6} \\
f\left(g_{1}, g_{2}\right) f\left(g_{3}, g_{3}\right) & =\left(-\frac{a_{11}}{2 b_{13}} \sqrt{2}\right)\left(a_{33}+d_{33} \sqrt{6}\right) \\
& =-\frac{a_{11} a_{33}}{2 b_{13}} \sqrt{2}-\frac{a_{11} d_{33}}{b_{13}} \sqrt{3} ;
\end{aligned}
$$

from $(28) f\left(g_{2}, g_{3}\right)^{g_{1}} f\left(g_{1}, g_{1}\right)=f\left(g_{1}, g_{2}\right) f\left(g_{3}, g_{3}\right)$ one obtains that $a_{23}=d_{23}=0$, $b_{23}=\frac{a_{33}}{2 b_{13}}, c_{23}=-\frac{d_{33}}{b_{13}}$. Thus $f\left(g_{2}, g_{3}\right)=\frac{a_{33}}{2 b_{13}} \sqrt{2}-\frac{d_{33}}{b_{13}} \sqrt{3}$.

$$
\text { Since } \begin{aligned}
f\left(g_{3}, g_{2}\right)^{g_{1}} f\left(g_{1}, g_{1}\right) & =\left(-\frac{a_{22} b_{13}}{a_{11}} \sqrt{2}\right)^{g_{1}} a_{11} \\
& =a_{22} b_{13} \sqrt{2} ;
\end{aligned}
$$

$f\left(g_{1}, g_{3}\right) f\left(g_{2}, g_{2}\right)=\left(b_{13} \sqrt{2}\right) a_{22}$ from $(31) f\left(g_{3}, g_{2}\right)^{g_{1}} f\left(g_{1}, g_{1}\right)=f\left(g_{1}, g_{3}\right) f\left(g_{2}, g_{2}\right)$ one obtains the identity $a_{22} b_{13} \sqrt{2}=a_{22} b_{13} \sqrt{2}$.

$$
\begin{aligned}
& \text { Since } f\left(g_{3}, g_{3}\right)^{g_{1}}=\left(a_{33}+d_{33} \sqrt{6}\right)^{g_{1}}=a_{33}-d_{33} \sqrt{6} ; \\
& f\left(g_{1}, g_{3}\right) f\left(g_{2}, g_{3}\right)=b_{13} \sqrt{2}\left(\frac{a_{33}}{2 b_{13}} \sqrt{2}-\frac{d_{33}}{b_{13}} \sqrt{3}\right)=a_{33}-d_{33} \sqrt{6} ;
\end{aligned}
$$

from $(32) f\left(g_{3}, g_{3}\right)^{g_{1}}=f\left(g_{1}, g_{3}\right) f\left(g_{2}, g_{3}\right)$ it is obtained the identity $a_{33}-d_{33} \sqrt{6}=a_{33}-d_{33} \sqrt{6}$.

$$
\text { Since } \begin{aligned}
f\left(g_{1}, g_{2}\right)^{g_{2}} f\left(g_{2}, g_{3}\right) & =\left(-\frac{a_{11}}{2 b_{31}} \sqrt{2}\right)^{g_{2}}\left(\frac{a_{33}}{2 b_{13}} \sqrt{2}-\frac{d_{33}}{b_{13}} \sqrt{3}\right) \\
& =-\frac{a_{11} a_{33}}{2 b_{13}^{2}}+\frac{a_{11} d_{33}}{2 b_{13}^{2}} \sqrt{6} ; \\
f\left(g_{2}, g_{1}\right) f\left(g_{3}, g_{2}\right) & =\left(a_{21}+b_{21} \sqrt{2}+c_{21} \sqrt{3}+d_{21} \sqrt{6}\right)\left(-\frac{a_{22} b_{13}}{a_{11}} \sqrt{2}\right) \\
& =-\frac{2 b_{21} a_{22} b_{13}}{a_{11}}-\frac{a_{21} a_{22} b_{13}}{a_{11}} \sqrt{2}-\frac{2 d_{21} a_{22} b_{13}}{a_{11}} \sqrt{3} \\
& -\frac{c_{21} a_{22} b_{13}}{a_{11}} \sqrt{6}
\end{aligned}
$$

from $(39) f\left(g_{1}, g_{2}\right)^{g_{2}} f\left(g_{2}, g_{3}\right)=f\left(g_{2}, g_{1}\right) f\left(g_{3}, g_{2}\right)$ one obtains that

$$
a_{21}=d_{21}=0, b_{21}=\frac{a_{11}^{2} a_{33}}{4 b_{13}^{3} a_{22}} \text { and } c_{21}=-\frac{a_{11}^{2} d_{33}}{2 b_{13}^{3} a_{22}} .
$$

Thus $f\left(g_{2}, g_{1}\right)=b_{21} \sqrt{2}+c_{21} \sqrt{3}$. From (4.5) $a_{31}=d_{31}=0$, so that $f\left(g_{3}, g_{1}\right)=b_{31} \sqrt{2}+c_{31} \sqrt{3}$.

$$
\begin{aligned}
\text { Since } f\left(g_{1}, g_{1}\right)^{g_{1}} & =a_{11} \\
f\left(g_{2}, g_{1}\right) f\left(g_{3}, g_{1}\right) & =\left(b_{21} \sqrt{2}+c_{21} \sqrt{3}\right)\left(b_{31} \sqrt{2}+c_{31} \sqrt{3}\right) \\
& =\left(2 b_{21} b_{31}+3 c_{21} c_{31}\right)+\left(b_{21} c_{31}+c_{21} b_{31}\right) \sqrt{6}
\end{aligned}
$$

from $(38) f\left(g_{1}, g_{1}\right)^{g_{1}}=f\left(g_{2}, g_{1}\right) f\left(g_{3}, g_{1}\right)$ one obtains the system

$$
\left\{\begin{array}{l}
2 b_{21} b_{31}+3 c_{21} c_{31}=a_{11} \\
b_{21} c_{31}+c_{21} b_{31}=0
\end{array}\right.
$$

Since $f\left(g_{1}, g_{3}\right)^{g_{2}} f\left(g_{2}, g_{2}\right)=b_{13} a_{22} \sqrt{2}$;

$$
\begin{aligned}
f\left(g_{2}, g_{1}\right) f\left(g_{3}, g_{3}\right) & =\left(b_{21} \sqrt{2}+c_{21} \sqrt{3}\right)\left(a_{33}+d_{33} \sqrt{6}\right) \\
& =\left(b_{21} a_{33}+3 c_{21} d_{33}\right) \sqrt{2}+\left(2 b_{21} d_{33}+c_{21} a_{33}\right) \sqrt{3}
\end{aligned}
$$


from $(40) f\left(g_{1}, g_{3}\right)^{g_{2}} f\left(g_{2}, g_{2}\right)=f\left(g_{2}, g_{1}\right) f\left(g_{3}, g_{3}\right)$ one obtains the system

$$
\left\{\begin{array}{l}
b_{21} a_{33}+3 c_{21} d_{33}=b_{13} a_{22} \\
2 b_{21} d_{33}+c_{21} a_{33}=0
\end{array}\right.
$$

Since $f\left(g_{3}, g_{1}\right)^{g_{2}} f\left(g_{2}, g_{2}\right)=\left(b_{31} \sqrt{2}-c_{31} \sqrt{3}\right) a_{22}=b_{31} a_{22} \sqrt{2}-c_{31} a_{22} \sqrt{3}$;

$$
f\left(g_{2}, g_{3}\right) f\left(g_{1}, g_{1}\right)=\left(\frac{a_{33}}{2 b_{13}} \sqrt{2}-\frac{d_{33}}{b_{13}} \sqrt{3}\right) a_{11}=\frac{a_{33} a_{11}}{2 b_{13}} \sqrt{2}-\frac{d_{33} a_{11}}{b_{13}} \sqrt{3}
$$

from $(46) f\left(g_{3}, g_{1}\right)^{g_{2}} f\left(g_{2}, g_{2}\right)=f\left(g_{2}, g_{3}\right) f\left(g_{1}, g_{1}\right)$ one obtains that

$$
b_{31}=\frac{a_{33} a_{11}}{2 a_{22} b_{13}}, c_{31}=\frac{d_{33} a_{11}}{a_{22} b_{13}} .
$$

$$
\text { Since } \begin{aligned}
f\left(g_{1}, g_{3}\right)^{g_{3}} f\left(g_{3}, g_{2}\right) & =\left(-b_{13} \sqrt{2}\right)\left(-\frac{a_{22} b_{13}}{a_{11}} \sqrt{2}\right)=2 \frac{a_{22} b_{13}^{2}}{a_{11}} ; \\
f\left(g_{3}, g_{1}\right) f\left(g_{2}, g_{3}\right) & =\left(b_{31} \sqrt{2}+c_{31} \sqrt{3}\right)\left(\frac{a_{33}}{2 b_{13}} \sqrt{2}-\frac{d_{33}}{b_{13}} \sqrt{3}\right) \\
& =\left(\frac{b_{31} a_{33}}{b_{13}}-3 \frac{c_{31} d_{33}}{b_{13}}\right)+\left(-\frac{b_{31} d_{33}}{b_{13}}+\frac{c_{31} a_{33}}{2 b_{13}}\right) \sqrt{6} ;
\end{aligned}
$$

from $(56) f\left(g_{1}, g_{3}\right)^{g_{3}} f\left(g_{3}, g_{2}\right)=f\left(g_{3}, g_{1}\right) f\left(g_{2}, g_{3}\right)$ one obtains

$$
\left\{\begin{array}{l}
b_{31} a_{33}-3 c_{31} d_{33}=2 \frac{a_{22} b_{13}^{3}}{a_{11}} \\
-b_{31} d_{33}+\frac{c_{31} a_{33}}{2}=0 .
\end{array}\right.
$$

Recalling (4.6) : $b_{21}=\frac{a_{11}^{2} a_{33}}{4 b_{13}^{3} a_{22}}, c_{21}=-\frac{a_{11}^{2} d_{33}}{2 b_{13}^{3} a_{22}}$.

From (4.8) $b_{31}=\frac{a_{33} a_{11}}{2 a_{22} b_{13}}, c_{31}=\frac{d_{33} a_{11}}{a_{22} b_{13}}$. If one replaces these values in the system (4.7)

$$
\left\{\begin{array}{l}
2 b_{21} b_{31}+3 c_{21} c_{31}=a_{11} \\
b_{21} c_{31}+c_{21} b_{31}=0
\end{array}\right.
$$

one obtains the main equation

$$
\frac{a_{11}^{2} a_{33}^{2}}{4 b_{13}^{4} a_{22}^{2}}-\frac{3}{2} \frac{a_{11}^{2} d_{33}^{2}}{b_{13}^{4} a_{22}^{2}}=1
$$

If $d_{33}=0, a_{33}= \pm \frac{2 b_{13}^{2} a_{22}}{a_{11}}, c_{21}=c_{31}=0$.

By taking $a_{33}=\frac{2 b_{13}^{2} a_{22}}{a_{11}} ; b_{21}=\frac{a_{11}}{2 b_{13}}, b_{31}=b_{13}$.

Thus, the matrix

$$
\left(\begin{array}{ccc}
f\left(g_{1}, g_{1}\right) & f\left(g_{1}, g_{2}\right) & f\left(g_{1}, g_{3}\right) \\
f\left(g_{2}, g_{1}\right) & f\left(g_{2}, g_{2}\right) & f\left(g_{2}, g_{3}\right) \\
f\left(g_{3}, g_{1}\right) & f\left(g_{3}, g_{2}\right) & f\left(g_{3}, g_{3}\right)
\end{array}\right)
$$

is

$$
\left(\begin{array}{ccc}
a_{11} & -\frac{a_{11}}{2 b_{13}} \sqrt{2} & b_{13} \sqrt{2} \\
\frac{a_{11}}{2 b_{13}} \sqrt{2} & a_{22} & \frac{b_{13} a_{22}}{a_{11}} \sqrt{2} \\
b_{13} \sqrt{2} & -\frac{a_{22} b_{13}}{a_{11}} \sqrt{2} & \frac{2 b_{13}^{2} a_{22}}{a_{11}}
\end{array}\right) .
$$


If $a_{11}=b_{13}=a_{22}=1$, one obtains the matrix

$$
\left(\begin{array}{ccc}
1 & -\frac{1}{2} \sqrt{2} & \sqrt{2} \\
\frac{1}{2} \sqrt{2} & 1 & \sqrt{2} \\
\sqrt{2} & -\sqrt{2} & 2
\end{array}\right)
$$

Therefore, the bilinear map $f$ such that

$$
[f]_{\mathcal{B}}=\left(\begin{array}{cccc} 
& & & \\
1 & 1 & 1 & 1 \\
1 & 1 & -\frac{1}{2} \sqrt{2} & \sqrt{2} \\
1 & \frac{1}{2} \sqrt{2} & 1 & \sqrt{2} \\
1 & \sqrt{2} & -\sqrt{2} & 2
\end{array}\right)
$$

is a nontrivial 2 -cocycle such that $\frac{\mathbb{Q}[X]}{\langle P\rangle} \rtimes_{f} G_{4}$ is a crossed product, where $P=X^{4}-5 X^{2}+6$ and $G_{4}$ is the Galois group of $P$ over $\mathbb{Q}$.

4.3.2. Cocycles for a crossed product $\frac{\mathbb{Q}[X]}{\langle P\rangle} \rtimes_{f} C_{2}$. Let $M=\mathbb{Q}(\sqrt{2}, \sqrt{3})$ and $K=\mathbb{Q}$. According to Fraleigh [8, Example 53.3] it is known that $M$ is Galois extension of $\mathbb{Q}$. Thus $M^{*}=M \backslash\{0\}$.

Since $M=\left\{a_{1}+a_{2} \sqrt{2}+a_{3} \sqrt{3}+a_{4} \sqrt{6} \mid a_{1}, a_{2}, a_{3}, a_{4} \in \mathbb{Q}\right\}, C_{2}=\{1, g\}=\langle g\rangle$ is described by

\begin{tabular}{r|cc} 
& 1 & $g$ \\
\hline 1 & 1 & 1 \\
$\sqrt{2}$ & $\sqrt{2}$ & $\sqrt{2}$ \\
$\sqrt{3}$ & $\sqrt{3}$ & $-\sqrt{3}$ \\
$\sqrt{6}$ & $\sqrt{6}$ & $-\sqrt{6}$
\end{tabular}

It is considered $\sigma: C_{2} \rightarrow A u t(M), g^{i} \mapsto \sigma\left(g^{i}\right): M \rightarrow M$ defined by $\sigma\left(g^{i}\right)\left(a_{1}+a_{2} \sqrt{2}+a_{3} \sqrt{3}+a_{4} \sqrt{6}\right)=\left(a_{1}+a_{2} \sqrt{2}+a_{3} \sqrt{3}+a_{4} \sqrt{6}\right)^{g^{i}}$.

Recalls that $C_{2} \times C_{2}=\{(1,1),(1, g),(g, 1),(g, g)\}$.

If $\left(g^{i}, g^{j}\right) \in C_{2} \times C_{2}$, then $\sigma\left(g^{i} g^{j}\right)=\sigma\left(g^{i}\right) \sigma\left(g^{j}\right)$.

In fact, if $a_{1}+a_{2} \sqrt{2}+a_{3} \sqrt{3}+a_{4} \sqrt{6} \in M$, then by (4.9):

$$
\begin{aligned}
\sigma(g g)\left(a_{1}+a_{2} \sqrt{2}+a_{3} \sqrt{3}+a_{4} \sqrt{6}\right) & =\sigma(1)\left(a_{1}+a_{2} \sqrt{2}+a_{3} \sqrt{3}+a_{4} \sqrt{6}\right) \\
& =a_{1}+a_{2} \sqrt{2}+a_{3} \sqrt{3}+a_{4} \sqrt{6}, \\
\sigma(g) \sigma(g)\left(a_{1}+a_{2} \sqrt{2}+a_{3} \sqrt{3}+a_{4} \sqrt{6}\right) & =\sigma(g)\left(a_{1}+a_{2} \sqrt{2}-a_{3} \sqrt{3}-a_{4} \sqrt{6}\right) \\
& =a_{1}+a_{2} \sqrt{2}+a_{3} \sqrt{3}+a_{4} \sqrt{6} .
\end{aligned}
$$

The others equalities are verified easily.

The map $\sigma(g)$ is a $K$-algebra automorphism of $M$.

If $\lambda \in K, a_{1}+a_{2} \sqrt{2}+a_{3} \sqrt{3}+a_{4} \sqrt{6}$ and $b_{1}+b_{2} \sqrt{2}+b_{3} \sqrt{3}+b_{4} \sqrt{6} \in M$, then :

1) $\sigma(g)\left(\lambda\left(a_{1}+a_{2} \sqrt{2}+a_{3} \sqrt{3}+a_{4} \sqrt{6}\right)\right)=\lambda \sigma(g)\left(a_{1}+a_{2} \sqrt{2}+a_{3} \sqrt{3}+a_{4} \sqrt{6}\right)$,

2) $\sigma(g)\left(\left(a_{1}+a_{2} \sqrt{2}+a_{3} \sqrt{3}+a_{4} \sqrt{6}\right)+\left(b_{1}+b_{2} \sqrt{2}+b_{3} \sqrt{3}+b_{4} \sqrt{6}\right)\right)$

$=\sigma(g)\left(a_{1}+a_{2} \sqrt{2}+a_{3} \sqrt{3}+a_{4} \sqrt{6}\right)+\sigma(g)\left(b_{1}+b_{2} \sqrt{2}+b_{3} \sqrt{3}+b_{4} \sqrt{6}\right)$,

3) $\sigma(g)\left(\left(a_{1}+a_{2} \sqrt{2}+a_{3} \sqrt{3}+a_{4} \sqrt{6}\right)\left(b_{1}+b_{2} \sqrt{2}+b_{3} \sqrt{3}+b_{4} \sqrt{6}\right)\right)$

$=\sigma(g)\left(a_{1}+a_{2} \sqrt{2}+a_{3} \sqrt{3}+a_{4} \sqrt{6}\right) \sigma(g)\left(b_{1}+b_{2} \sqrt{2}+b_{3} \sqrt{3}+b_{4} \sqrt{6}\right)$.

Therefore, $C_{2}$ acts (weakly) on $M$.

Example 4.1. Find the value of $\alpha \in M^{*}$ such that

$$
f\left(g^{i}, g^{j}\right)=\left\{\begin{array}{cc}
1 & \text { if } i+j<2 \\
\alpha & \text { if } i+j \geq 2
\end{array}\right.
$$


where $0 \leq i, j<2 ; g^{i}, g^{j} \in C_{2}=\{1, g\}=\langle g\rangle$ and $f: C_{2} \times C_{2} \rightarrow M^{*}$ is a 2-cocycle of the crossed product $M \rtimes_{f} C_{2}$.

Since $\alpha \in M^{*}$, then $\alpha=a+b \sqrt{2}+c \sqrt{3}+d \sqrt{6}$ where $a^{2}+b^{2}+c^{2}+d^{2} \neq 0$. From the definition of crossed product, the following three conditions are satisfied :

1) $f\left(1, g^{i}\right)=f\left(g^{i}, 1\right)=1$,

2) $f\left(g^{j}, g^{k}\right)^{g^{i}} f\left(g^{i}, g^{j+k}\right)=f\left(g^{i}, g^{j}\right) f\left(g^{i+j}, g^{k}\right)$,

3) $\left(\left(b_{1}+b_{2} \sqrt{2}+b_{3} \sqrt{3}+b_{4} \sqrt{6}\right)^{g^{j}}\right)^{g^{i}} f\left(g^{i}, g^{j}\right)=f\left(g^{i}, g^{j}\right)\left(b_{1}+b_{2} \sqrt{2}+b_{3} \sqrt{3}+b_{4} \sqrt{6}\right)^{g^{i+j}}$, where $b_{1}+b_{2} \sqrt{2}+b_{3} \sqrt{3}+b_{4} \sqrt{6} \in M$.

$$
\begin{aligned}
& C_{2} \times C_{2} \times C_{2}=\{1, g\} \times\{1, g\} \times\{1, g\} \\
= & \{(1,1,1),(1,1, g),(1, g, 1),(1, g, g),(g, 1,1),(g, 1, g),(g, g, 1),(g, g, g)\} .
\end{aligned}
$$

Considering this set the assertion 2) for $f, f\left(g^{j}, g^{k}\right)^{g^{i}} f\left(g^{i}, g^{j+k}\right)=f\left(g^{i}, g^{j}\right) f\left(g^{i+j}, g^{k}\right)$, is expanded to the following 8 equalities :
(1) $1=f(1,1)^{1} f(1,(1)(1))=f(1,1) f((1)(1), 1)$,
(2) $1=f(1, g)^{1} f(1,(1)(g))=f(1,1) f((1)(1), g)$,
(3) $1=f(g, 1)^{1} f(1,(g)(1))=f(1, g) f((1)(g), 1)$,
(4) $\alpha=f(g, g)^{1} f(1,(g)(g))=f(1, g) f((1)(g), g)$,
(5) $1=f(1,1)^{g} f(g,(1)(1))=f(g, 1) f((g)(1), 1)$,
(6) $\alpha=f(1, g)^{g} f(g,(1)(g))=f(g, 1) f((g)(1), g)$,
(7) $\alpha=f(g, 1)^{g} f(g,(g)(1))=f(g, g) f((g)(g), 1)$,
(8) $\alpha^{g}=f(g, g)^{g} f\left(g, g^{2}\right)=f(g, g) f\left(g^{2}, g\right)=\alpha$,

where $\alpha=a+b \sqrt{2}+c \sqrt{3}+d \sqrt{6}, \alpha^{g}=a+b \sqrt{2}-c \sqrt{3}-d \sqrt{6}$.

From the equality (8), it is deduced that $\alpha=a+b \sqrt{2} \square$

Setting $a=3 \& b=5$ is obtained the matrix

$$
(3+5 \sqrt{2})
$$

Therefore, the bilinear map $f$ such that

$$
[f]_{\mathcal{B}}=\left(\begin{array}{cc}
1 & 1 \\
1 & 3+5 \sqrt{2}
\end{array}\right)
$$

is a nontrivial 2-cocycle such that $A \rtimes_{f} C_{2}$ is a crossed product, where $A=\frac{\mathbb{Q}[X]}{\left\langle X^{4}-5 X^{2}+6\right\rangle}$ and $C_{2}$ is the subgroup $\langle g\rangle$ of the Galois group of $X^{4}-5 X^{2}+6$ over $\mathbb{Q}$.

4.4. Cocycles for a crossed product $\frac{\mathbb{Q}[X]}{\langle P\rangle} \rtimes_{f} G_{6}$. Let $\zeta=\zeta_{3}=-\frac{1}{2}+\frac{\sqrt{3}}{2} i$ be the primitive $3-$ th root of the unity in the field of complex numbers $\mathbb{C}$. Let be $M=\mathbb{Q}\left(\sqrt[3]{2}, \zeta_{3}\right), K=\mathbb{Q}$ and $G(M / K)$ is the Galois group of $M$ over $K$. According to [8, Example 50.9] it is known that $M$ is Galois extension of $\mathbb{Q}$. The proof of [8, Theorem 31.4] then shows that $\left\{1, \sqrt[3]{2}, \sqrt[3]{2^{2}}, \zeta, \zeta \sqrt[3]{2}, \zeta \sqrt[3]{2^{2}}\right\}$ is a basis for $M$ over $\mathbb{Q}$. Thus $M=\left\{a_{1}+a_{2} \sqrt[3]{2}+a_{3} \sqrt[3]{2^{2}}+a_{4} \zeta+a_{5} \zeta \sqrt[3]{2}+a_{6} \zeta \sqrt[3]{2^{2}} \mid a_{i} \in \mathbb{Q}\right\}$. Then $G(M / K)=\left\{g_{0}, g_{1}, g_{2}, g_{3}, g_{4}, g_{5}\right\}$ is described as follows

\begin{tabular}{r|cccccc} 
& $g_{0}$ & $g_{1}$ & $g_{2}$ & $g_{3}$ & $g_{4}$ & $g_{5}$ \\
\hline 1 & 1 & 1 & 1 & 1 & 1 & 1 \\
$\sqrt[3]{2}$ & $\sqrt[3]{2}$ & $\zeta \sqrt[3]{2}$ & $\zeta^{2} \sqrt[3]{2}$ & $\sqrt[3]{2}$ & $\zeta \sqrt[3]{2}$ & $\zeta^{2} \sqrt[3]{2}$ \\
$\sqrt[3]{2^{2}}$ & $\sqrt[3]{2^{2}}$ & $\zeta^{2} \sqrt[3]{2^{2}}$ & $\zeta \sqrt[3]{2^{2}}$ & $\sqrt[3]{2^{2}}$ & $\zeta^{2} \sqrt[3]{2^{2}}$ & $\zeta \sqrt[3]{2^{2}}$ \\
$\zeta$ & $\zeta$ & $\zeta$ & $\zeta$ & $\zeta^{2}$ & $\zeta^{2}$ & $\zeta^{2}$ \\
$\zeta \sqrt[3]{2}$ & $\zeta \sqrt[3]{2}$ & $\zeta^{2} \sqrt[3]{2}$ & $\sqrt[3]{2}$ & $\zeta^{2} \sqrt[3]{2}$ & $\sqrt[3]{2}$ & $\zeta \sqrt[3]{2}$ \\
$\zeta \sqrt[3]{2^{2}}$ & $\zeta \sqrt[3]{2^{2}}$ & $\sqrt[3]{2^{2}}$ & $\zeta^{2} \sqrt[3]{2^{2}}$ & $\zeta^{2} \sqrt[3]{2^{2}}$ & $\zeta \sqrt[3]{2^{2}}$ & $\sqrt[3]{2^{2}}$
\end{tabular}


Hence, the group table for $G(M / K)$ is given by

\begin{tabular}{c|llllll} 
& $g_{0}$ & $g_{1}$ & $g_{2}$ & $g_{3}$ & $g_{4}$ & $g_{5}$ \\
\hline$g_{0}$ & $g_{0}$ & $g_{1}$ & $g_{2}$ & $g_{3}$ & $g_{4}$ & $g_{5}$ \\
$g_{1}$ & $g_{1}$ & $g_{2}$ & $g_{0}$ & $g_{4}$ & $g_{5}$ & $g_{3}$ \\
$g_{2}$ & $g_{2}$ & $g_{0}$ & $g_{1}$ & $g_{5}$ & $g_{3}$ & $g_{4}$ \\
$g_{3}$ & $g_{3}$ & $g_{5}$ & $g_{4}$ & $g_{0}$ & $g_{2}$ & $g_{1}$ \\
$g_{4}$ & $g_{4}$ & $g_{3}$ & $g_{5}$ & $g_{1}$ & $g_{0}$ & $g_{2}$ \\
$g_{5}$ & $g_{5}$ & $g_{4}$ & $g_{3}$ & $g_{2}$ & $g_{1}$ & $g_{0}$.
\end{tabular}

It is considered $\sigma: G(M / K) \rightarrow \operatorname{Aut}(M), g_{i} \mapsto \sigma\left(g_{i}\right): M \rightarrow M$ defined by

$\sigma\left(g_{i}\right)\left(a_{1}+a_{2} \sqrt[3]{2}+a_{3} \sqrt[3]{2^{2}}+a_{4} \zeta+a_{5} \zeta \sqrt[3]{2}+a_{6} \zeta \sqrt[3]{2^{2}}\right)=\left(a_{1}+a_{2} \sqrt[3]{2}+a_{3} \sqrt[3]{2^{2}}+a_{4} \zeta+a_{5} \zeta \sqrt[3]{2}+a_{6} \zeta \sqrt[3]{2^{2}}\right)^{g_{i}}$.

Recalls that

$G(M / K) \times G(M / K)=\left\{\left(g_{0}, g_{0}\right),\left(g_{0}, g_{1}\right),\left(g_{0}, g_{2}\right),\left(g_{0}, g_{3}\right),\left(g_{0}, g_{4}\right),\left(g_{0}, g_{5}\right), \ldots\right.$,

$\left.\left(g_{5}, g_{0}\right),\left(g_{5}, g_{1}\right),\left(g_{5}, g_{2}\right),\left(g_{5}, g_{3}\right),\left(g_{5}, g_{4}\right),\left(g_{5}, g_{5}\right)\right\}$.

If $\left(g_{i}, g_{j}\right) \in G(M / K) \times G(M / K)$, then $\sigma\left(g_{i} g_{j}\right)=\sigma\left(g_{i}\right) \sigma\left(g_{j}\right)$.

In fact, if $a=a_{1}+a_{2} \sqrt[3]{2}+a_{3} \sqrt[3]{2^{2}}+a_{4} \zeta+a_{5} \zeta \sqrt[3]{2}+a_{6} \zeta \sqrt[3]{2^{2}} \in M$, then by (4.11) \& (4.12):

$$
\begin{aligned}
\sigma\left(g_{1} g_{2}\right)(a) & =\sigma\left(g_{0}\right)(a)=a \\
\sigma\left(g_{1}\right) \sigma\left(g_{2}\right)(a) & =\sigma\left(g_{1}\right)\left(a_{1}+a_{2} \zeta^{2} \sqrt[3]{2}+a_{3} \zeta \sqrt[3]{2^{2}}+a_{4} \zeta+a_{5} \sqrt[3]{2}+a_{6} \zeta^{2} \sqrt[3]{2^{2}}\right)=a .
\end{aligned}
$$

The others equalities are checked similarly.

Since $G(M / K)$ is the Galois group of $M$ over $K$, the map $\sigma\left(g_{i}\right)$ is a $K$-algebra automorphism of $M$. That is; if $\lambda \in K, b=b_{1}+b_{2} \sqrt[3]{2}+b_{3} \sqrt[3]{2^{2}}+b_{4} \zeta+b_{5} \zeta \sqrt[3]{2}+b_{6} \zeta \sqrt[3]{2^{2}} \in M$, then :

$$
\begin{aligned}
& \text { 1) } \sigma\left(g_{i}\right)(\lambda a)=\lambda \sigma\left(g_{i}\right)(a), \\
& \text { 2) } \sigma\left(g_{i}\right)(a+b)=\sigma\left(g_{i}\right)(a)+\sigma\left(g_{i}\right)(b), \\
& \text { 3) } \sigma\left(g_{i}\right)(a b)=\sigma\left(g_{i}\right)(a) \sigma\left(g_{i}\right)(b) .
\end{aligned}
$$

Therefore, $G(M / K)$ acts (weakly) on $M$.

By the primitive element theorem [2, p 74] exists $\gamma \in M$ such that $M=K[\gamma]=\frac{K[X]}{\langle\operatorname{irr}(\gamma, K)\rangle}$ is a field; so the group of units of $M$ is $M^{*}=M \backslash\{0\}$.

Let $f: G(M / K) \times G(M / K) \rightarrow M^{*}$ be a function such that $E=M \rtimes_{f} G(M / K)$ is a crossed product. Then the following three conditions are satisfied according to Blattner, Cohen, and Montgomery [3, Corollary 4.6] :

1) $f\left(g_{0}, g_{i}\right)=f\left(g_{i}, g_{0}\right)=1$,

2) $f\left(g_{j}, g_{k}\right)^{g_{i}} f\left(g_{i}, g_{j} g_{k}\right)=f\left(g_{i}, g_{j}\right) f\left(g_{i} g_{j}, g_{k}\right)$,

3) $\left(b^{g_{j}}\right)^{g_{i}} f\left(g_{i}, g_{j}\right)=f\left(g_{i}, g_{j}\right) b^{g_{i} g_{j}}$, where $b \in M$.

The set $G(M / K) \times G(M / K) \times G(M / K)$ has $6^{3}=216$ elements .

The assertion 3$)$ for $f,\left(b^{g_{j}}\right)^{g_{i}} f\left(g_{i}, g_{j}\right)=f\left(g_{i}, g_{j}\right) b^{g_{i} g_{j}}$, is satisfied due to that the $K$-algebra $M$ is commutative and $G(M / K) \times M \rightarrow M,\left(g_{i}, a\right) \mapsto a^{g_{i}}$ is an action of $G(M / K)$ on $M$.

Using the group table (4.12), $f\left(g_{j}, g_{k}\right)^{g_{i}} f\left(g_{i}, g_{j} g_{k}\right)=f\left(g_{i}, g_{j}\right) f\left(g_{i} g_{j}, g_{k}\right)$ is expanded to 216 equalities. Since $f\left(g_{j}, g_{k}\right)^{g_{0}} f\left(g_{0}, g_{j} g_{k}\right)=f\left(g_{0}, g_{j}\right) f\left(g_{j}, g_{k}\right) ; f\left(g_{0}, g_{k}\right)^{g_{i}} f\left(g_{i}, g_{k}\right)=f\left(g_{i}, g_{0}\right) f\left(g_{i}, g_{k}\right)$ and $f\left(g_{j}, g_{0}\right)^{g_{i}} f\left(g_{i}, g_{j}\right)=f\left(g_{i}, g_{j}\right) f\left(g_{i} g_{j}, g_{0}\right)$; the following 125 equalities hold :

(44) $f\left(g_{1}, g_{1}\right)^{g_{1}} f\left(g_{1}, g_{2}\right)=f\left(g_{1}, g_{1}\right) f\left(g_{2}, g_{1}\right)$,

(45) $f\left(g_{1}, g_{2}\right)^{g_{1}}=f\left(g_{1}, g_{1}\right) f\left(g_{2}, g_{2}\right)$,

(46) $f\left(g_{1}, g_{3}\right)^{g_{1}} f\left(g_{1}, g_{4}\right)=f\left(g_{1}, g_{1}\right) f\left(g_{2}, g_{3}\right)$,

(47) $f\left(g_{1}, g_{4}\right)^{g_{1}} f\left(g_{1}, g_{5}\right)=f\left(g_{1}, g_{1}\right) f\left(g_{2}, g_{4}\right)$,

(48) $f\left(g_{1}, g_{5}\right)^{g_{1}} f\left(g_{1}, g_{3}\right)=f\left(g_{1}, g_{1}\right) f\left(g_{2}, g_{5}\right)$,

(50) $f\left(g_{2}, g_{1}\right)^{g_{1}}=f\left(g_{1}, g_{2}\right)$,

(51) $f\left(g_{2}, g_{2}\right)^{g_{1}} f\left(g_{1}, g_{1}\right)=f\left(g_{1}, g_{2}\right)$,

(52) $f\left(g_{2}, g_{3}\right)^{g_{1}} f\left(g_{1}, g_{5}\right)=f\left(g_{1}, g_{2}\right)$,

(53) $f\left(g_{2}, g_{4}\right)^{g_{1}} f\left(g_{1}, g_{3}\right)=f\left(g_{1}, g_{2}\right)$,
(54) $f\left(g_{2}, g_{5}\right)^{g_{1}} f\left(g_{1}, g_{4}\right)=f\left(g_{1}, g_{2}\right)$,

(56) $f\left(g_{3}, g_{1}\right)^{g_{1}} f\left(g_{1}, g_{5}\right)=f\left(g_{1}, g_{3}\right) f\left(g_{4}, g_{1}\right)$,

(57) $f\left(g_{3}, g_{2}\right)^{g_{1}} f\left(g_{1}, g_{4}\right)=f\left(g_{1}, g_{3}\right) f\left(g_{4}, g_{2}\right)$,

(58) $f\left(g_{3}, g_{3}\right)^{g_{1}}=f\left(g_{1}, g_{3}\right) f\left(g_{4}, g_{3}\right)$,

(59) $f\left(g_{3}, g_{4}\right)^{g_{1}} f\left(g_{1}, g_{2}\right)=f\left(g_{1}, g_{3}\right) f\left(g_{4}, g_{4}\right)$,

(60) $f\left(g_{3}, g_{5}\right)^{g_{1}} f\left(g_{1}, g_{1}\right)=f\left(g_{1}, g_{3}\right) f\left(g_{4}, g_{5}\right)$,

(62) $f\left(g_{4}, g_{1}\right)^{g_{1}} f\left(g_{1}, g_{3}\right)=f\left(g_{1}, g_{4}\right) f\left(g_{5}, g_{1}\right)$,

(63) $f\left(g_{4}, g_{2}\right)^{g_{1}} f\left(g_{1}, g_{5}\right)=f\left(g_{1}, g_{4}\right) f\left(g_{5}, g_{2}\right)$,

(64) $f\left(g_{4}, g_{3}\right)^{g_{1}} f\left(g_{1}, g_{1}\right)=f\left(g_{1}, g_{4}\right) f\left(g_{5}, g_{3}\right)$, 
(65) $f\left(g_{4}, g_{4}\right)^{g_{1}}=f\left(g_{1}, g_{4}\right) f\left(g_{5}, g_{4}\right)$,

(66) $f\left(g_{4}, g_{5}\right)^{g_{1}} f\left(g_{1}, g_{2}\right)=f\left(g_{1}, g_{4}\right) f\left(g_{5}, g_{5}\right)$,

(68) $f\left(g_{5}, g_{1}\right)^{g_{1}} f\left(g_{1}, g_{4}\right)=f\left(g_{1}, g_{5}\right) f\left(g_{3}, g_{1}\right)$,

(69) $f\left(g_{5}, g_{2}\right)^{g_{1}} f\left(g_{1}, g_{3}\right)=f\left(g_{1}, g_{5}\right) f\left(g_{3}, g_{2}\right)$,

(70) $f\left(g_{5}, g_{3}\right)^{g_{1}} f\left(g_{1}, g_{2}\right)=f\left(g_{1}, g_{5}\right) f\left(g_{3}, g_{3}\right)$,

(71) $f\left(g_{5}, g_{4}\right)^{g_{1}} f\left(g_{1}, g_{1}\right)=f\left(g_{1}, g_{5}\right) f\left(g_{3}, g_{4}\right)$,

(72) $f\left(g_{5}, g_{5}\right)^{g_{1}}=f\left(g_{1}, g_{5}\right) f\left(g_{3}, g_{5}\right)$,

(80) $f\left(g_{1}, g_{1}\right)^{g_{2}} f\left(g_{2}, g_{2}\right)=f\left(g_{2}, g_{1}\right) f\left(g_{0}, g_{1}\right)$,

(81) $f\left(g_{1}, g_{2}\right)^{g_{2}}=f\left(g_{2}, g_{1}\right)$,

(82) $f\left(g_{1}, g_{3}\right)^{g_{2}} f\left(g_{2}, g_{4}\right)=f\left(g_{2}, g_{1}\right)$,

(83) $f\left(g_{1}, g_{4}\right)^{g_{2}} f\left(g_{2}, g_{5}\right)=f\left(g_{2}, g_{1}\right)$,

(84) $f\left(g_{1}, g_{5}\right)^{g_{2}} f\left(g_{2}, g_{3}\right)=f\left(g_{2}, g_{1}\right)$,

(86) $f\left(g_{2}, g_{1}\right)^{g_{2}}=f\left(g_{2}, g_{2}\right) f\left(g_{1}, g_{1}\right)$,

(87) $f\left(g_{2}, g_{2}\right)^{g_{2}} f\left(g_{2}, g_{1}\right)=f\left(g_{2}, g_{2}\right) f\left(g_{1}, g_{2}\right)$,

(88) $f\left(g_{2}, g_{3}\right)^{g_{2}} f\left(g_{2}, g_{5}\right)=f\left(g_{2}, g_{2}\right) f\left(g_{1}, g_{3}\right)$,

(89) $f\left(g_{2}, g_{4}\right)^{g_{2}} f\left(g_{2}, g_{3}\right)=f\left(g_{2}, g_{2}\right) f\left(g_{1}, g_{4}\right)$,

(90) $f\left(g_{2}, g_{5}\right)^{g_{2}} f\left(g_{2}, g_{4}\right)=f\left(g_{2}, g_{2}\right) f\left(g_{1}, g_{5}\right)$,

(92) $f\left(g_{3}, g_{1}\right)^{g_{2}} f\left(g_{2}, g_{5}\right)=f\left(g_{2}, g_{3}\right) f\left(g_{5}, g_{1}\right)$,

(93) $f\left(g_{3}, g_{2}\right)^{g_{2}} f\left(g_{2}, g_{4}\right)=f\left(g_{2}, g_{3}\right) f\left(g_{5}, g_{2}\right)$,

(94) $f\left(g_{3}, g_{3}\right)^{g_{2}}=f\left(g_{2}, g_{3}\right) f\left(g_{5}, g_{3}\right)$,

(95) $f\left(g_{3}, g_{4}\right)^{g_{2}} f\left(g_{2}, g_{2}\right)=f\left(g_{2}, g_{3}\right) f\left(g_{5}, g_{4}\right)$,

(96) $f\left(g_{3}, g_{5}\right)^{g_{2}} f\left(g_{2}, g_{1}\right)=f\left(g_{2}, g_{3}\right) f\left(g_{5}, g_{5}\right)$,

(98) $f\left(g_{4}, g_{1}\right)^{g_{2}} f\left(g_{2}, g_{3}\right)=f\left(g_{2}, g_{4}\right) f\left(g_{3}, g_{1}\right)$,

(99) $f\left(g_{4}, g_{2}\right)^{g_{2}} f\left(g_{2}, g_{5}\right)=f\left(g_{2}, g_{4}\right) f\left(g_{3}, g_{2}\right)$,

(100) $f\left(g_{4}, g_{3}\right)^{g_{2}} f\left(g_{2}, g_{1}\right)=f\left(g_{2}, g_{4}\right) f\left(g_{3}, g_{3}\right)$,

(101) $f\left(g_{4}, g_{4}\right)^{g_{2}}=f\left(g_{2}, g_{4}\right) f\left(g_{3}, g_{4}\right)$,

(102) $f\left(g_{4}, g_{5}\right)^{g_{2}} f\left(g_{2}, g_{2}\right)=f\left(g_{2}, g_{4}\right) f\left(g_{3}, g_{5}\right)$,

(104) $f\left(g_{5}, g_{1}\right)^{g_{2}} f\left(g_{2}, g_{4}\right)=f\left(g_{2}, g_{5}\right) f\left(g_{4}, g_{1}\right)$,

(105) $f\left(g_{5}, g_{2}\right)^{g_{2}} f\left(g_{2}, g_{3}\right)=f\left(g_{2}, g_{5}\right) f\left(g_{4}, g_{2}\right)$,

(106) $f\left(g_{5}, g_{3}\right)^{g_{2}} f\left(g_{2}, g_{2}\right)=f\left(g_{2}, g_{5}\right) f\left(g_{4}, g_{3}\right)$,

(107) $f\left(g_{5}, g_{4}\right)^{g_{2}} f\left(g_{2}, g_{1}\right)=f\left(g_{2}, g_{5}\right) f\left(g_{4}, g_{4}\right)$,

(108) $f\left(g_{5}, g_{5}\right)^{g_{2}}=f\left(g_{2}, g_{5}\right) f\left(g_{4}, g_{5}\right)$,

(116) $f\left(g_{1}, g_{1}\right)^{g_{3}} f\left(g_{3}, g_{2}\right)=f\left(g_{3}, g_{1}\right) f\left(g_{5}, g_{1}\right)$,

(117) $f\left(g_{1}, g_{2}\right)^{g_{3}}=f\left(g_{3}, g_{1}\right) f\left(g_{5}, g_{2}\right)$,

(118) $f\left(g_{1}, g_{3}\right)^{g_{3}} f\left(g_{3}, g_{4}\right)=f\left(g_{3}, g_{1}\right) f\left(g_{5}, g_{3}\right)$,

(119) $f\left(g_{1}, g_{4}\right)^{g_{3}} f\left(g_{3}, g_{5}\right)=f\left(g_{3}, g_{1}\right) f\left(g_{5}, g_{4}\right)$,

(120) $f\left(g_{1}, g_{5}\right)^{g_{3}} f\left(g_{3}, g_{3}\right)=f\left(g_{3}, g_{1}\right) f\left(g_{5}, g_{5}\right)$,

(122) $f\left(g_{2}, g_{1}\right)^{g_{3}}=f\left(g_{3}, g_{2}\right) f\left(g_{4}, g_{1}\right)$,

(123) $f\left(g_{2}, g_{2}\right)^{g_{3}} f\left(g_{3}, g_{1}\right)=f\left(g_{3}, g_{2}\right) f\left(g_{4}, g_{2}\right)$,

(124) $f\left(g_{2}, g_{3}\right)^{g_{3}} f\left(g_{3}, g_{5}\right)=f\left(g_{3}, g_{2}\right) f\left(g_{4}, g_{3}\right)$,

(125) $f\left(g_{2}, g_{4}\right)^{g_{3}} f\left(g_{3}, g_{3}\right)=f\left(g_{3}, g_{2}\right) f\left(g_{4}, g_{4}\right)$,

(126) $f\left(g_{2}, g_{5}\right)^{g_{3}} f\left(g_{3}, g_{4}\right)=f\left(g_{3}, g_{2}\right) f\left(g_{4}, g_{5}\right)$,

(128) $f\left(g_{3}, g_{1}\right)^{g_{3}} f\left(g_{3}, g_{5}\right)=f\left(g_{3}, g_{3}\right)$,

(129) $f\left(g_{3}, g_{2}\right)^{g_{3}} f\left(g_{3}, g_{4}\right)=f\left(g_{3}, g_{3}\right)$,

(130) $f\left(g_{3}, g_{3}\right)^{g_{3}}=f\left(g_{3}, g_{3}\right)$,

(131) $f\left(g_{3}, g_{4}\right)^{g_{3}} f\left(g_{3}, g_{2}\right)=f\left(g_{3}, g_{3}\right)$,

(132) $f\left(g_{3}, g_{5}\right)^{g_{3}} f\left(g_{3}, g_{1}\right)=f\left(g_{3}, g_{3}\right)$,

(134) $f\left(g_{4}, g_{1}\right)^{g_{3}} f\left(g_{3}, g_{3}\right)=f\left(g_{3}, g_{4}\right) f\left(g_{2}, g_{1}\right)$,

(135) $f\left(g_{4}, g_{2}\right)^{g_{3}} f\left(g_{3}, g_{5}\right)=f\left(g_{3}, g_{4}\right) f\left(g_{2}, g_{2}\right)$,

(136) $f\left(g_{4}, g_{3}\right)^{g_{3}} f\left(g_{3}, g_{1}\right)=f\left(g_{3}, g_{4}\right) f\left(g_{2}, g_{3}\right)$,

(137) $f\left(g_{4}, g_{4}\right)^{g_{3}}=f\left(g_{3}, g_{4}\right) f\left(g_{2}, g_{4}\right)$,

(138) $f\left(g_{4}, g_{5}\right)^{g_{3}} f\left(g_{3}, g_{2}\right)=f\left(g_{3}, g_{4}\right) f\left(g_{2}, g_{5}\right)$,

(140) $f\left(g_{5}, g_{1}\right)^{g_{3}} f\left(g_{3}, g_{4}\right)=f\left(g_{3}, g_{5}\right) f\left(g_{1}, g_{1}\right)$,

(141) $f\left(g_{5}, g_{2}\right)^{g_{3}} f\left(g_{3}, g_{3}\right)=f\left(g_{3}, g_{5}\right) f\left(g_{1}, g_{2}\right)$,
(142) $f\left(g_{5}, g_{3}\right)^{g_{3}} f\left(g_{3}, g_{2}\right)=f\left(g_{3}, g_{5}\right) f\left(g_{1}, g_{3}\right)$,

(143) $f\left(g_{5}, g_{4}\right)^{g_{3}} f\left(g_{2}, g_{1}\right)=f\left(g_{3}, g_{5}\right) f\left(g_{1}, g_{4}\right)$,

(144) $f\left(g_{5}, g_{5}\right)^{g_{3}}=f\left(g_{3}, g_{5}\right) f\left(g_{1}, g_{5}\right)$,

(152) $f\left(g_{1}, g_{1}\right)^{g_{4}} f\left(g_{4}, g_{2}\right)=f\left(g_{4}, g_{1}\right) f\left(g_{3}, g_{1}\right)$,

(153) $f\left(g_{1}, g_{2}\right)^{g_{4}}=f\left(g_{4}, g_{1}\right) f\left(g_{3}, g_{2}\right)$,

(154) $f\left(g_{1}, g_{3}\right)^{g_{4}} f\left(g_{4}, g_{4}\right)=f\left(g_{4}, g_{1}\right) f\left(g_{3}, g_{3}\right)$,

(155) $f\left(g_{1}, g_{4}\right)^{g_{4}} f\left(g_{4}, g_{5}\right)=f\left(g_{4}, g_{1}\right) f\left(g_{3}, g_{4}\right)$,

(156) $f\left(g_{1}, g_{5}\right)^{g_{4}} f\left(g_{4}, g_{3}\right)=f\left(g_{4}, g_{1}\right) f\left(g_{3}, g_{5}\right)$,

(158) $f\left(g_{2}, g_{1}\right)^{g_{4}}=f\left(g_{4}, g_{2}\right) f\left(g_{5}, g_{1}\right)$,

(159) $f\left(g_{2}, g_{2}\right)^{g_{4}} f\left(g_{4}, g_{1}\right)=f\left(g_{4}, g_{2}\right) f\left(g_{5}, g_{2}\right)$,

(160) $f\left(g_{2}, g_{3}\right)^{g_{4}} f\left(g_{4}, g_{5}\right)=f\left(g_{4}, g_{2}\right) f\left(g_{5}, g_{3}\right)$,

(161) $f\left(g_{2}, g_{4}\right)^{g_{4}} f\left(g_{4}, g_{3}\right)=f\left(g_{4}, g_{2}\right) f\left(g_{5}, g_{4}\right)$,

(162) $f\left(g_{2}, g_{5}\right)^{g_{4}} f\left(g_{4}, g_{4}\right)=f\left(g_{4}, g_{2}\right) f\left(g_{5}, g_{5}\right)$,

(164) $f\left(g_{3}, g_{1}\right)^{g_{4}} f\left(g_{4}, g_{5}\right)=f\left(g_{4}, g_{3}\right) f\left(g_{1}, g_{1}\right)$,

(165) $f\left(g_{3}, g_{2}\right)^{g_{4}} f\left(g_{4}, g_{4}\right)=f\left(g_{4}, g_{3}\right) f\left(g_{1}, g_{2}\right)$,

(166) $f\left(g_{3}, g_{3}\right)^{g_{4}}=f\left(g_{4}, g_{3}\right) f\left(g_{1}, g_{3}\right)$,

(167) $f\left(g_{3}, g_{4}\right)^{g_{4}} f\left(g_{4}, g_{2}\right)=f\left(g_{4}, g_{3}\right) f\left(g_{1}, g_{4}\right)$,

(168) $f\left(g_{3}, g_{5}\right)^{g_{4}} f\left(g_{4}, g_{1}\right)=f\left(g_{4}, g_{3}\right) f\left(g_{1}, g_{5}\right)$,

(170) $f\left(g_{4}, g_{1}\right)^{g_{4}} f\left(g_{4}, g_{3}\right)=f\left(g_{4}, g_{4}\right)$,

(171) $f\left(g_{4}, g_{2}\right)^{g_{4}} f\left(g_{4}, g_{5}\right)=f\left(g_{4}, g_{4}\right)$,

(172) $f\left(g_{4}, g_{3}\right)^{g_{4}} f\left(g_{4}, g_{1}\right)=f\left(g_{4}, g_{4}\right)$,

(173) $f\left(g_{4}, g_{4}\right)^{g_{4}}=f\left(g_{4}, g_{4}\right)$,

(174) $f\left(g_{4}, g_{5}\right)^{g_{4}} f\left(g_{4}, g_{2}\right)=f\left(g_{4}, g_{4}\right)$,

(176) $f\left(g_{5}, g_{1}\right)^{g_{4}} f\left(g_{4}, g_{4}\right)=f\left(g_{4}, g_{5}\right) f\left(g_{2}, g_{1}\right)$,

(177) $f\left(g_{5}, g_{2}\right)^{g_{4}} f\left(g_{4}, g_{3}\right)=f\left(g_{4}, g_{5}\right) f\left(g_{2}, g_{2}\right)$,

(178) $f\left(g_{5}, g_{3}\right)^{g_{4}} f\left(g_{4}, g_{2}\right)=f\left(g_{4}, g_{5}\right) f\left(g_{2}, g_{3}\right)$,

(179) $f\left(g_{5}, g_{4}\right)^{g_{4}} f\left(g_{4}, g_{1}\right)=f\left(g_{4}, g_{5}\right) f\left(g_{2}, g_{4}\right)$,

(180) $f\left(g_{5}, g_{5}\right)^{g_{4}}=f\left(g_{4}, g_{5}\right) f\left(g_{2}, g_{5}\right)$,

(188) $f\left(g_{1}, g_{1}\right)^{g_{5}} f\left(g_{5}, g_{2}\right)=f\left(g_{5}, g_{1}\right) f\left(g_{4}, g_{1}\right)$,

(189) $f\left(g_{1}, g_{2}\right)^{g_{5}}=f\left(g_{5}, g_{1}\right) f\left(g_{4}, g_{2}\right)$,

(190) $f\left(g_{1}, g_{3}\right)^{g_{5}} f\left(g_{5}, g_{4}\right)=f\left(g_{5}, g_{1}\right) f\left(g_{4}, g_{3}\right)$,

(191) $f\left(g_{1}, g_{4}\right)^{g_{5}} f\left(g_{5}, g_{5}\right)=f\left(g_{5}, g_{1}\right) f\left(g_{4}, g_{4}\right)$,

(192) $f\left(g_{1}, g_{5}\right)^{g_{5}} f\left(g_{5}, g_{3}\right)=f\left(g_{5}, g_{1}\right) f\left(g_{4}, g_{5}\right)$,

(194) $f\left(g_{2}, g_{1}\right)^{g_{5}}=f\left(g_{5}, g_{2}\right) f\left(g_{3}, g_{1}\right)$,

(195) $f\left(g_{2}, g_{2}\right)^{g_{5}} f\left(g_{5}, g_{1}\right)=f\left(g_{5}, g_{2}\right) f\left(g_{3}, g_{2}\right)$,

(196) $f\left(g_{2}, g_{3}\right)^{g_{5}} f\left(g_{5}, g_{5}\right)=f\left(g_{5}, g_{2}\right) f\left(g_{3}, g_{3}\right)$,

(197) $f\left(g_{2}, g_{4}\right)^{g_{5}} f\left(g_{5}, g_{3}\right)=f\left(g_{5}, g_{2}\right) f\left(g_{3}, g_{4}\right)$,

(198) $f\left(g_{2}, g_{5}\right)^{g_{5}} f\left(g_{5}, g_{4}\right)=f\left(g_{5}, g_{2}\right) f\left(g_{3}, g_{5}\right)$,

(200) $f\left(g_{3}, g_{1}\right)^{g_{5}} f\left(g_{5}, g_{5}\right)=f\left(g_{5}, g_{3}\right) f\left(g_{2}, g_{1}\right)$,

(201) $f\left(g_{3}, g_{2}\right)^{g_{5}} f\left(g_{5}, g_{4}\right)=f\left(g_{5}, g_{3}\right) f\left(g_{2}, g_{2}\right)$,

(202) $f\left(g_{3}, g_{3}\right)^{g_{5}}=f\left(g_{5}, g_{3}\right) f\left(g_{2}, g_{3}\right)$,

(203) $f\left(g_{3}, g_{4}\right)^{g_{5}} f\left(g_{5}, g_{2}\right)=f\left(g_{5}, g_{3}\right) f\left(g_{2}, g_{4}\right)$,

(204) $f\left(g_{3}, g_{5}\right)^{g_{5}} f\left(g_{5}, g_{1}\right)=f\left(g_{5}, g_{3}\right) f\left(g_{2}, g_{5}\right)$,

(206) $f\left(g_{4}, g_{1}\right)^{g_{5}} f\left(g_{5}, g_{3}\right)=f\left(g_{5}, g_{4}\right) f\left(g_{1}, g_{1}\right)$,

(207) $f\left(g_{4}, g_{2}\right)^{g_{5}} f\left(g_{5}, g_{5}\right)=f\left(g_{5}, g_{4}\right) f\left(g_{1}, g_{2}\right)$,

(208) $f\left(g_{4}, g_{3}\right)^{g_{5}} f\left(g_{5}, g_{1}\right)=f\left(g_{5}, g_{4}\right) f\left(g_{1}, g_{3}\right)$,

(209) $f\left(g_{4}, g_{4}\right)^{g_{5}}=f\left(g_{5}, g_{4}\right) f\left(g_{1}, g_{4}\right)$,

(210) $f\left(g_{4}, g_{5}\right)^{g_{5}} f\left(g_{5}, g_{2}\right)=f\left(g_{5}, g_{4}\right) f\left(g_{1}, g_{5}\right)$,

(212) $f\left(g_{5}, g_{1}\right)^{g_{5}} f\left(g_{5}, g_{4}\right)=f\left(g_{5}, g_{5}\right)$,

(213) $f\left(g_{5}, g_{2}\right)^{g_{5}} f\left(g_{5}, g_{3}\right)=f\left(g_{5}, g_{5}\right)$,

(214) $f\left(g_{5}, g_{3}\right)^{g_{5}} f\left(g_{5}, g_{2}\right)=f\left(g_{5}, g_{5}\right)$,

(215) $f\left(g_{5}, g_{4}\right)^{g_{5}} f\left(g_{5}, g_{1}\right)=f\left(g_{5}, g_{5}\right)$,

(216) $f\left(g_{5}, g_{5}\right)^{g_{5}}=f\left(g_{5}, g_{5}\right)$,

If $f\left(g_{i}, g_{j}\right)=a_{i j}+b_{i j} \sqrt[3]{2}+c_{i j} \sqrt[3]{2^{2}}+d_{i j} \zeta+e_{i j} \zeta \sqrt[3]{2}+k_{i j} \zeta \sqrt[3]{2^{2}}, f\left(g_{3}, g_{3}\right)^{g_{3}}=\left(a_{33}-d_{33}\right)+\left(b_{33}-\right.$ $\left.e_{33}\right) \sqrt[3]{2}+\left(c_{33}-k_{33}\right) \sqrt[3]{2^{2}}-d_{33} \zeta-e_{33} \zeta \sqrt[3]{2}-k_{33} \zeta \sqrt[3]{2^{2}}$. From $f\left(g_{3}, g_{3}\right)^{g_{3}}=f\left(g_{3}, g_{3}\right)$, it follows that $f\left(g_{3}, g_{3}\right)=a_{33}+b_{33} \sqrt[3]{2}+c_{33} \sqrt[3]{2^{2}}$.

Of course, by applying the condition of normality 1$)$ of $f$, one may know that exist 25 variables $f\left(g_{i}, g_{j}\right)$ for $i, j: 1,2,3,4,5$. Furthermore, each of these variables depends on 6 variables. Thus, exist 150 variables. 
That is, one has a system of 125 equations in 150 variables. It is noted that the equations (128) with (132), (129) with (131), (170) with (172), (171) with (174), (212) with (215) \& (213) with (214) are the same. So, the system is reduced to one of 119 equations in 150 variables.

By following the procedure applied in computing nonrational 2 -cocycles, recalling that $(a+b \sqrt[3]{2}+$ $\left.c \sqrt[3]{2^{2}}+d \zeta+e \zeta \sqrt[3]{2}+f \zeta \sqrt[3]{2^{2}}\right)\left(a^{\prime}+b^{\prime} \sqrt[3]{2}+c^{\prime} \sqrt[3]{2^{2}}+d^{\prime} \zeta+e^{\prime} \zeta \sqrt[3]{2}+f^{\prime} \zeta \sqrt[3]{2^{2}}\right)=\left(a a^{\prime}+2 b c^{\prime}+2 c b^{\prime}-\right.$ $\left.d d^{\prime}-2 e f^{\prime}-2 f e^{\prime}\right)+\left(a b^{\prime}+b a^{\prime}+2 c c^{\prime}-d e^{\prime}-e d^{\prime}-2 f f^{\prime}\right) \sqrt[3]{2}+\left(a c^{\prime}+b b^{\prime}+c a^{\prime}-d f^{\prime}-e e^{\prime}-f d^{\prime}\right) \sqrt[3]{2^{2}}+$ $\left(a d^{\prime}+2 b f^{\prime}+2 c e^{\prime}+d\left(a^{\prime}-d^{\prime}\right)+2 e\left(c^{\prime}-f^{\prime}\right)+2 f\left(b^{\prime}-e^{\prime}\right)\right) \zeta+\left(a e^{\prime}+b d^{\prime}+2 c f^{\prime}+d\left(b^{\prime}-e^{\prime}\right)+e\left(a^{\prime}-\right.\right.$ $\left.\left.d^{\prime}\right)+2 f\left(c^{\prime}-f^{\prime}\right)\right) \zeta \sqrt[3]{2}+\left(a f^{\prime}+b e^{\prime}+c d^{\prime}+d\left(c^{\prime}-f^{\prime}\right)+e\left(b^{\prime}-c^{\prime}\right)+f\left(a^{\prime}-d^{\prime}\right)\right) \zeta \sqrt[3]{2^{2}}$ and resolving the system of equations, one can obtain the cocycles $f$.

Then the matrix on $\mathbb{Q}$

$$
\left(\begin{array}{lllll}
f\left(g_{1}, g_{1}\right) & f\left(g_{1}, g_{2}\right) & f\left(g_{1}, g_{3}\right) & f\left(g_{1}, g_{4}\right) & f\left(g_{1}, g_{5}\right) \\
f\left(g_{2}, g_{1}\right) & f\left(g_{2}, g_{2}\right) & f\left(g_{2}, g_{3}\right) & f\left(g_{2}, g_{4}\right) & f\left(g_{2}, g_{5}\right) \\
f\left(g_{3}, g_{1}\right) & f\left(g_{3}, g_{2}\right) & f\left(g_{3}, g_{3}\right) & f\left(g_{3}, g_{4}\right) & f\left(g_{3}, g_{5}\right) \\
f\left(g_{4}, g_{1}\right) & f\left(g_{4}, g_{2}\right) & f\left(g_{4}, g_{3}\right) & f\left(g_{4}, g_{4}\right) & f\left(g_{4}, g_{5}\right) \\
f\left(g_{5}, g_{1}\right) & f\left(g_{5}, g_{2}\right) & f\left(g_{5}, g_{3}\right) & f\left(g_{5}, g_{4}\right) & f\left(g_{5}, g_{5}\right)
\end{array}\right)
$$

can be written as

$$
\left(\begin{array}{ccccc}
a_{11} & a_{11} a_{22} & a_{13} & \frac{a_{11}^{2} a_{22}}{a_{13} a_{15}} & a_{15} \\
a_{11} a_{22} & a_{22} & \frac{a_{11} a_{22}}{a_{15}} & \frac{a_{11} a_{22}}{a_{13}} & \frac{a_{13} a_{15}}{a_{11}} \\
\frac{a_{11}^{4} a_{22}^{2} a_{33}^{2}}{a_{13}^{4} a_{15} a_{44}^{2}} & \frac{a_{11} a_{22} a_{33}}{a_{13} a_{44}} & a_{33} & \frac{a_{13} a_{44}}{a_{11} a_{22}} & \frac{a_{15} a_{33}^{2}}{a_{13}^{2} a_{44}} \\
\frac{a_{13} a_{44}}{a_{33}} & \frac{a_{11}^{3} a_{22}^{2} a_{33}}{a_{13}^{3} a_{15} a_{44}} & \frac{a_{33}}{a_{13}} & a_{44} & \frac{a_{13}^{3} a_{15} a_{44}^{2}}{a_{11}^{3} a_{22}^{2} a_{33}} \\
\frac{a_{13}^{3} a_{15} a_{44}}{a_{11}^{2} a_{22} a_{33}} & \frac{a_{13}^{4} a_{15} a_{44}^{2}}{a_{11}^{3} a_{22} a_{33}^{2}} & \frac{a_{15} a_{33}}{a_{11} a_{22}} & \frac{a_{13} a_{15} a_{44}}{a_{11}^{2} a_{22}} & \frac{a_{13}^{4} a_{15}^{2} a_{44}^{2}}{a_{11}^{4} a_{22}^{2} a_{33}}
\end{array}\right)
$$

where $a_{13}^{6} a_{44}^{3}=a_{11}^{4} a_{22}^{2} a_{33}^{3}$ ( from of (56) and (200)) .

This matrix presents a solution of the above system of 119 equations in 150 variables. By setting $a_{33}=a_{44}=3, a_{11}=a_{13}=a_{22}=2 \& a_{15}=2$, one obtains the matrix

$$
\left(\begin{array}{ccccc}
2 & 4 & 2 & 2 & 2 \\
4 & 2 & 2 & 2 & 2 \\
2 & 2 & 3 & \frac{3}{2} & \frac{3}{2} \\
2 & 2 & \frac{3}{2} & 3 & \frac{3}{2} \\
2 & 2 & \frac{3}{2} & \frac{3}{2} & 3
\end{array}\right)
$$

Therefore, the rational bilinear form $f$ such that

$$
[f]_{\mathcal{B}}=\left(\begin{array}{cccccc}
1 & 1 & 1 & 1 & 1 & 1 \\
1 & 2 & 4 & 2 & 2 & 2 \\
1 & 4 & 2 & 2 & 2 & 2 \\
1 & 2 & 2 & 3 & \frac{3}{2} & \frac{3}{2} \\
1 & 2 & 2 & \frac{3}{2} & 3 & \frac{3}{2} \\
1 & 2 & 2 & \frac{3}{2} & \frac{3}{2} & 3
\end{array}\right)
$$

is a required nontrivial 2-cocycle such that $A \rtimes_{f} G$ is a crossed product, where $A=\frac{\mathbb{Q}[X]}{\left\langle X^{3}-2\right\rangle}$ and $G=S_{3}$ is the Galois group of $X^{3}-2$ over $\mathbb{Q}$.

4.5. Cocycle for a crossed product $\frac{\mathbb{Q}[X]}{\langle P\rangle} \rtimes_{f} G_{8}$. Let $\zeta=\zeta_{4}=i$ be the primitive $4-$ th root of the unity in the field of complex numbers $\mathbb{C}$. Let be $M=\mathbb{Q}\left(\sqrt[4]{2}, \zeta_{4}\right), K=\mathbb{Q}$ and $G(M / K)$ is the Galois 
group of $M$ over $K$. According to [8, Example 50.9] it is known that $M$ is Galois extension of $\mathbb{Q}$.

The proof of [8, Theorem 31.4] then shows that $\left\{1, \sqrt[4]{2}, \sqrt[4]{2^{2}}, \sqrt[4]{2^{3}}, \zeta, \zeta \sqrt[4]{2}, \zeta \sqrt[4]{2^{2}}, \zeta \sqrt[4]{2^{3}}\right\}$ is a basis for $M$ over $\mathbb{Q}$. Thus $M=\left\{a_{1}+a_{2} \sqrt[4]{2}+a_{3} \sqrt[4]{2^{2}}+a_{4} \sqrt[4]{2^{3}}+a_{5} \zeta+a_{6} \zeta \sqrt[4]{2}+a_{7} \zeta \sqrt[4]{2^{2}}+a_{8} \zeta \sqrt[4]{2^{3}} \mid a_{i} \in \mathbb{Q}\right\}$. Then $G(M / K)=\left\{g_{0}, g_{1}, g_{2}, g_{3}, g_{4}, g_{5}, g_{6}, g_{7}\right\}$ is described as follows

(4.14)

\begin{tabular}{r|cccccccc} 
& $g_{0}$ & $g_{1}$ & $g_{2}$ & $g_{3}$ & $g_{4}$ & $g_{5}$ & $g_{6}$ & $g_{7}$ \\
\hline 1 & 1 & 1 & 1 & 1 & 1 & 1 & 1 & 1 \\
$\sqrt[4]{2}$ & $\sqrt[4]{2}$ & $\zeta \sqrt[4]{2}$ & $\zeta^{2} \sqrt[4]{2}$ & $\zeta^{3} \sqrt[4]{2}$ & $\sqrt[4]{2}$ & $\zeta \sqrt[4]{2}$ & $\zeta^{2} \sqrt[4]{2}$ & $\zeta^{3} \sqrt[4]{2}$ \\
$\sqrt[4]{2^{2}}$ & $\sqrt[4]{2^{2}}$ & $\zeta^{2} \sqrt[4]{2^{2}}$ & $\sqrt[4]{2^{2}}$ & $\zeta^{2} \sqrt[4]{2^{2}}$ & $\sqrt[4]{2^{2}}$ & $\zeta^{2} \sqrt[4]{2^{2}}$ & $\sqrt[4]{2^{2}}$ & $\zeta^{2} \sqrt[4]{2^{2}}$ \\
$\sqrt[4]{2^{3}}$ & $\sqrt[4]{2^{3}}$ & $\zeta^{3} \sqrt[4]{2^{3}}$ & $\zeta^{2} \sqrt[4]{2^{3}}$ & $\zeta \sqrt[4]{2^{3}}$ & $\sqrt[4]{2^{3}}$ & $\zeta^{3} \sqrt[4]{2^{3}}$ & $\zeta^{2} \sqrt[4]{2^{3}}$ & $\zeta \sqrt[4]{2^{3}}$ \\
$\zeta$ & $\zeta$ & $\zeta$ & $\zeta$ & $\zeta$ & $-\zeta$ & $-\zeta$ & $-\zeta$ & $-\zeta$ \\
$\zeta \sqrt[4]{2}$ & $\zeta \sqrt[4]{2}$ & $\zeta^{2} \sqrt[4]{2}$ & $\zeta^{3} \sqrt[4]{2}$ & $\sqrt[4]{2}$ & $-\zeta \sqrt[4]{2}$ & $-\zeta^{2} \sqrt[4]{2}$ & $-\zeta^{3} \sqrt[4]{2}$ & $-\sqrt[4]{2}$ \\
$\zeta \sqrt[4]{2^{2}}$ & $\zeta \sqrt[4]{2^{2}}$ & $\zeta^{3} \sqrt[4]{2^{2}}$ & $\zeta \sqrt[4]{2^{2}}$ & $\zeta^{3} \sqrt[4]{2^{2}}$ & $-\zeta \sqrt[4]{2^{2}}$ & $-\zeta^{3} \sqrt[4]{2^{2}}$ & $-\zeta \sqrt[4]{2^{2}}$ & $-\zeta^{3} \sqrt[4]{2^{2}}$ \\
$\zeta \sqrt[4]{2^{3}}$ & $\zeta \sqrt[4]{2^{3}}$ & $\sqrt[4]{2^{3}}$ & $\zeta^{3} \sqrt[4]{2^{3}}$ & $\zeta^{2} \sqrt[4]{2^{3}}$ & $-\zeta \sqrt[4]{2^{3}}$ & $-\sqrt[4]{2^{3}}$ & $-\zeta^{3} \sqrt[4]{2^{3}}$ & $-\zeta^{2} \sqrt[4]{2^{3}}$
\end{tabular}

Hence, the group table for $G(M / K)$ is given by

\begin{tabular}{l|llllllll} 
& $g_{0}$ & $g_{1}$ & $g_{2}$ & $g_{3}$ & $g_{4}$ & $g_{5}$ & $g_{6}$ & $g_{7}$ \\
\hline$g_{0}$ & $g_{0}$ & $g_{1}$ & $g_{2}$ & $g_{3}$ & $g_{4}$ & $g_{5}$ & $g_{6}$ & $g_{7}$ \\
$g_{1}$ & $g_{1}$ & $g_{2}$ & $g_{3}$ & $g_{0}$ & $g_{5}$ & $g_{6}$ & $g_{7}$ & $g_{4}$ \\
$g_{2}$ & $g_{2}$ & $g_{3}$ & $g_{0}$ & $g_{1}$ & $g_{6}$ & $g_{7}$ & $g_{4}$ & $g_{5}$ \\
$g_{3}$ & $g_{3}$ & $g_{0}$ & $g_{1}$ & $g_{2}$ & $g_{7}$ & $g_{4}$ & $g_{5}$ & $g_{6}$ \\
$g_{4}$ & $g_{4}$ & $g_{7}$ & $g_{6}$ & $g_{5}$ & $g_{0}$ & $g_{3}$ & $g_{2}$ & $g_{1}$ \\
$g_{5}$ & $g_{5}$ & $g_{4}$ & $g_{7}$ & $g_{6}$ & $g_{1}$ & $g_{0}$ & $g_{3}$ & $g_{2}$ \\
$g_{6}$ & $g_{6}$ & $g_{5}$ & $g_{4}$ & $g_{7}$ & $g_{2}$ & $g_{1}$ & $g_{0}$ & $g_{3}$ \\
$g_{7}$ & $g_{7}$ & $g_{6}$ & $g_{5}$ & $g_{4}$ & $g_{3}$ & $g_{2}$ & $g_{1}$ & $g_{0}$.
\end{tabular}

Since $G(M / K)$ is the Galois group of $M$ over $K, G(M / K)$ acts (weakly) on $M$.

By the primitive element theorem exists $\gamma \in M$ such that $M=K[\gamma]=\frac{K[X]}{\langle\operatorname{irr}(\gamma, K)\rangle}$ is a field; so the group of units of $M$ is $M^{*}=M \backslash\{0\}$.

Let $f: G(M / K) \times G(M / K) \rightarrow M^{*}$ be a function such that $E=M \rtimes_{f} G(M / K)$ is a crossed product. Then the following three conditions are satisfied :

1) $f\left(g_{0}, g_{i}\right)=f\left(g_{i}, g_{0}\right)=1$,

2) $f\left(g_{j}, g_{k}\right)^{g_{i}} f\left(g_{i}, g_{j} g_{k}\right)=f\left(g_{i}, g_{j}\right) f\left(g_{i} g_{j}, g_{k}\right)$

3) $\left(b^{g_{j}}\right)^{g_{i}} f\left(g_{i}, g_{j}\right)=f\left(g_{i}, g_{j}\right) b^{g_{i} g_{j}}$, where $b \in M$.

The set $G(M / K) \times G(M / K) \times G(M / K)$ has $8^{3}=512$ elements. Using the group table (4.15), $f\left(g_{j}, g_{k}\right)^{g_{i}} f\left(g_{i}, g_{j} g_{k}\right)=f\left(g_{i}, g_{j}\right) f\left(g_{i} g_{j}, g_{k}\right)$ is expanded to 512 equalities.

Since the assertion 3) is satisfied clearly, assuming that the assertions 1) and 2) are satisfied, and applying the procedure developed in Subsection 4.3 one can obtain the family of 2 -cocycles $f$ for the crossed product $\frac{\mathbb{Q}[X]}{\left\langle X^{4}-2\right\rangle} \rtimes_{f} G_{8}$.

Thus, the matrix on $\mathbb{Q}$

$$
\left(\begin{array}{lllllll}
f\left(g_{1}, g_{1}\right) & f\left(g_{1}, g_{2}\right) & f\left(g_{1}, g_{3}\right) & f\left(g_{1}, g_{4}\right) & f\left(g_{1}, g_{5}\right) & f\left(g_{1}, g_{6}\right) & f\left(g_{1}, g_{7}\right) \\
f\left(g_{2}, g_{1}\right) & f\left(g_{2}, g_{2}\right) & f\left(g_{2}, g_{3}\right) & f\left(g_{2}, g_{4}\right) & f\left(g_{2}, g_{5}\right) & f\left(g_{2}, g_{6}\right) & f\left(g_{2}, g_{7}\right) \\
f\left(g_{3}, g_{1}\right) & f\left(g_{3}, g_{2}\right) & f\left(g_{3}, g_{3}\right) & f\left(g_{3}, g_{4}\right) & f\left(g_{3}, g_{5}\right) & f\left(g_{3}, g_{6}\right) & f\left(g_{3}, g_{7}\right) \\
f\left(g_{4}, g_{1}\right) & f\left(g_{4}, g_{2}\right) & f\left(g_{4}, g_{3}\right) & f\left(g_{4}, g_{4}\right) & f\left(g_{4}, g_{5}\right) & f\left(g_{4}, g_{6}\right) & f\left(g_{4}, g_{7}\right) \\
f\left(g_{5}, g_{1}\right) & f\left(g_{5}, g_{2}\right) & f\left(g_{5}, g_{3}\right) & f\left(g_{5}, g_{4}\right) & f\left(g_{5}, g_{5}\right) & f\left(g_{5}, g_{6}\right) & f\left(g_{5}, g_{7}\right) \\
f\left(g_{6}, g_{1}\right) & f\left(g_{6}, g_{2}\right) & f\left(g_{6}, g_{3}\right) & f\left(g_{6}, g_{4}\right) & f\left(g_{6}, g_{5}\right) & f\left(g_{6}, g_{6}\right) & f\left(g_{6}, g_{7}\right) \\
f\left(g_{7}, g_{1}\right) & f\left(g_{7}, g_{2}\right) & f\left(g_{7}, g_{3}\right) & f\left(g_{7}, g_{4}\right) & f\left(g_{7}, g_{5}\right) & f\left(g_{7}, g_{6}\right) & f\left(g_{7}, g_{7}\right)
\end{array}\right)
$$


can be written as

$$
\left(\begin{array}{ccccccc}
4 & \frac{1}{2} & \frac{1}{2} & 1 & 1 & 1 & 1 \\
\frac{1}{2} & \frac{1}{16} & \frac{1}{8} & \frac{1}{4} & \frac{1}{4} & \frac{1}{4} & \frac{1}{4} \\
\frac{1}{2} & \frac{1}{8} & 1 & \frac{1}{2} & \frac{1}{2} & \frac{1}{2} & \frac{1}{2} \\
1 & \frac{1}{4} & \frac{1}{2} & 1 & 2 & 4 & 1 \\
1 & \frac{1}{4} & \frac{1}{2} & 1 & 1 & 2 & 4 \\
1 & \frac{1}{4} & \frac{1}{2} & 4 & 1 & 1 & 2 \\
1 & \frac{1}{4} & \frac{1}{2} & 2 & 4 & 1 & 1
\end{array}\right) .
$$

Consequently, the rational bilinear form $f$ such that

$$
[f]_{\mathcal{B}}=\left(\begin{array}{cccccccc}
1 & 1 & 1 & 1 & 1 & 1 & 1 & 1 \\
1 & 4 & \frac{1}{2} & \frac{1}{2} & 1 & 1 & 1 & 1 \\
1 & \frac{1}{2} & \frac{1}{16} & \frac{1}{8} & \frac{1}{4} & \frac{1}{4} & \frac{1}{4} & \frac{1}{4} \\
1 & \frac{1}{2} & \frac{1}{8} & 1 & \frac{1}{2} & \frac{1}{2} & \frac{1}{2} & \frac{1}{2} \\
1 & 1 & \frac{1}{4} & \frac{1}{2} & 1 & 2 & 4 & 1 \\
1 & 1 & \frac{1}{4} & \frac{1}{2} & 1 & 1 & 2 & 4 \\
1 & 1 & \frac{1}{4} & \frac{1}{2} & 4 & 1 & 1 & 2 \\
1 & 1 & \frac{1}{4} & \frac{1}{2} & 2 & 4 & 1 & 1
\end{array}\right)
$$

is a nontrivial 2-cocycle such that $A \rtimes_{f} G_{8}$ is a crossed product, where $A=\frac{\mathbb{Q}[X]}{\left\langle X^{4}-2\right\rangle}$ and $G_{8}$ is the Galois group of $X^{4}-2$ over $\mathbb{Q}$.

\section{Conclusions.}

1. The cocycle $f$ of the crossed product $\frac{\mathbb{Q}[X]}{\langle P\rangle} \rtimes_{f} G_{4}$ can take values outside of $\mathbb{Q}$ because $\sqrt{2} \in \mathbb{Q}(\sqrt{2}, \sqrt{3}) \backslash \mathbb{Q}$ (Subsubsection 4.3.1).

2. The cocycle $f$ of the crossed product $\frac{\mathbb{Q}[X]}{\langle P\rangle} \rtimes_{f} C_{2}$ can take values outside of $\mathbb{Q}$ because $3+5 \sqrt{2} \in \mathbb{Q}(\sqrt{2}) \backslash \mathbb{Q}$, where $C_{2}=\langle g\rangle$ is a subgroup of $G_{4}$ (Example 4.1).

3. Considering $G=S_{3}$ as Galois group of the polynomial $X^{3}-2$ over $\mathbb{Q}, A$ as the $\mathbb{Q}$-algebra $\frac{\mathbb{Q}[X]}{\left\langle X^{3}-2\right\rangle}$, one can find a nontrivial 2-cocycle $f$ (see (4.13)) so that $A \rtimes_{f} G$ is a crossed product.

4. The problem of to find the family of rational 2-cocycles in order that $E=M \rtimes_{f} G(M / \mathbb{Q})$ to be crossed product, it is reduced to the resolution of the system of algebraic equations with several variables over the field $\mathbb{Q}$, which is determined with the condition of normality and the condition of 2-cocycle (Subsection 4.4).

6. Acknowledgements. This article was written while the author was in Lima-Peru with a License granted by the Universidad Nacional del Altiplano de Puno in order to publish in indexed journals.

The author gratefully thank Dr. Christian Valqui and Dr. Juan J. Guccione for providing him helpful suggestions, the bibliografic material and ideas used in this work. The author also thanks the Institute of Mathematics and Related Sciences (IMCA) for giving him the opportunity to investigate in the process of executing his doctoral thesis. The author also wish to thank Julio Gutiérrez for helpful comments.

ORCID and License

Felipe Clímaco Ccolque T. https:// orcid.org/0000-0002-9440-3569

This work is licensed under the Creative Commons - Attribution 4.0 International (CC BY 4.0)

\section{References}

[1] Guccione JA, Guccione JJ. The (co)homology of crossed products over monogenic algebras. East West J. of Mathematics. 2001; 3:49-67. 
[2] Ccolque Taipe F.C. Criterio de Galois para la Solubilidad por Radicales de Polinomios de grado $n \geq 5$. [Tesis de título]. Arequipa : Facultad de Ciencias Naturales y Formales, Universidad Nacional de San Agustín de Arequipa; 2000.

[3] Blattner RJ, Cohen M, and Montgomery S. Crossed products and inner actions of Hopf algebras. Trans. Amer. Math. Soc. 1986; 298:671-711.

[4] Hilgemann M.J. On Finite-dimensional Hopf Algebras and Their Classifications. [Thesis Ph.D]. Iowa: Iowa State University; 2010.

[5] Guccione JA, Guccione JJ. Hochschild (co)homology of Hopf crossed products. K-theory. 2002; 25(2):138-169.

[6] Hoffman K, Kunze R. Linear Algebra. 2nd ed. New Jersey : Prentice Hall, Inc.,Englewood Cliffs; 1971.

[7] Loday JL. Cyclic Homology. Berlin: Springer Verlag; 1998.

[8] Fraleigh JB. A first Course in Abstract Algebra. Reading: Addison Wesley; 1967.

[9] Hilton PJ, Stammbach U. A course in Homological Algebra. New York Heidelberg Berlin: Springer Verlag; 1971. 